\title{
Integral MRAC with minimal controller synthesis and bounded adaptive gains: the continuous-time case
}

\author{
Umberto Montanaro ${ }^{\mathrm{a}, *}$, Josep M. Olm ${ }^{\mathrm{b}}$ \\ ${ }^{a}$ Department of Industrial Engineering, University of Naples Federico II, Naples 80125, Italy \\ ${ }^{b}$ Department of Mathematics \& Institute of Industrial and Control Engineering, Universitat \\ Politècnica de Catalunya, 08028 Barcelona, Spain.
}

\begin{abstract}
Model reference adaptive controllers designed via the Minimal Control Synthesis (MCS) approach are a viable solution to control plants affected by parameter uncertainty, unmodelled dynamics, and disturbances. Despite its effectiveness to impose the required reference dynamics, an apparent drift of the adaptive gains, which can eventually lead to closed-loop instability or alter tracking performance, may occasionally be induced by external disturbances. This problem has been recently addressed for this class of adaptive algorithms in the discrete-time case and for square-integrable perturbations by using a parameter projection strategy [1]. In this paper we tackle systematically this issue for MCS continuous-time adaptive systems with integral action by enhancing the adaptive mechanism not only with a parameter projection method, but also embedding a $\sigma$-modification strategy. The former is used to preserve convergence to zero of the tracking error when the disturbance is bounded and $L_{2}$, while the latter guarantees global uniform ultimate boundedness under continuous $L_{\infty}$ disturbances. In both cases, the proposed control schemes ensure boundedness of all the closed-loop signals. The strategies are numerically validated by considering systems subject to different kinds of disturbances. In addition, an electrical power circuit is used to show the applicability of the algorithms to engineering problems requiring a precise tracking of a reference profile over a long time range despite disturbances, unmodelled dynamics, and parameter uncertainty.
\end{abstract}

Keywords: Continuous-time Model Reference Adaptive Control, Parameter

\footnotetext{
${ }^{*}$ Corresponding author: Umberto Montanaro

Email addresses: umberto.montanaro@unina.it (Umberto Montanaro), josep. olmeupc . edu (Josep M. Olm)
} 
projection, Switching $\sigma$-modification

\section{Introduction}

Adaptive control was introduced in the early 1950's to deal with systems that exhibit high levels of parametric uncertainty and variability [2]. Nowadays it is a subject of active research, with improvements of the original algorithms and combinations with other control techniques such as sliding modes, neural networks, iterative learning control, or fuzzy controllers (see for example $[3,4,5,6,7,8,9]$ and references therein) being periodically reported.

Model Reference Adaptive Control (MRAC) schemes with Minimal Controller Synthesis (MCS) [10,11] are effective strategies to impose the dynamics of a certain reference model to linear plants in control canonical form affected by unknown, uncertain or time-varying parameters, unmodelled nonlinear dynamics, and disturbances. Examples of applications where the MCS has been successfully used include electronic throttle valves [12, 13], common rail systems [14, 15], electromechanical valve actuators for future camless engines [16], synchronization of chaotic systems [17], shaking tables in civil engineering [18], active engine mounts [19], hydraulic test rigs [20], and cantilever beams [21].

Since its formulation in $[10,11]$, different enhancements of the original MCS algorithm have been proposed to tackle analytically both specific problems and tracking performance improvement. For example, multi-input continuous-time systems can be controlled via the decentralized MCS [22]; rapidly time-varying disturbances can be suppressed by the Extended MCS (EMCS), which embeds an additional switching action [23]; the Integral MCS (MCSI) [24] includes an integral control action where the integral gain is adaptive itself, and it is used to further improve tracking performance. Recently, MCS control schemes for piecewise affine systems have been proposed in [25, 26, 27]. The MCS algorithm for discrete-time systems has been presented in [28, 29], and extended to piecewise linear systems in [30]. In the discrete-time setting, integral and switching control actions have been proposed in [14] and [31], respectively.

However, in spite of the proved effectiveness of the MCS in a number of cases, an apparently unbounded drift of the adaptive gains has been occasionally observed (see, for example, [29, 14]), which may eventually yield to loss of tracking performance or even closed-loop instability. This phenomenon, also common to other MRAC schemes [32, 2], is due to the fact that, from a theoretical point 
of view, asymptotically zero tracking error plus closed-loop stability can be rigorously proved only for uncertain, Linear Time-Invariant (LTI) systems, while unmodelled dynamics and disturbances are tackled under rather tight assumptions [11] not always satisfied in practice. This issue cannot be fully addressed neither with the integral action of the MCSI, nor with the method introduced in [33], and represents a serious drawback of the MCS.

Recently, a parameter projection-based [2] scheme embedded in a discretetime MCS algorithm was capable of providing asymptotic tracking of the reference model and boundedness of the adaptive gains in the face of $L_{2}$ disturbances for uncertain discrete-time linear systems [1]. In this paper we present a continuous-time counterpart of this algorithm, and we also propose an alternative strategy to deal with generic bounded disturbances, guaranteeing in both cases boundedness of all the closed-loop signals, including the adaptive gains. Specifically, we first introduce parameter projection to cope with $L_{2} \cap L_{\infty}$ disturbances. This locking method allows to confine the adaptive gains to a preassigned set while ensuring asymptotically zero tracking error and closed-loop stability for square integrable disturbances. The resulting control algorithm is denoted in the paper as MCSI-PP (MCS with Integral control action and Parameter Projection). In turn, when the goal is to tame bounded disturbances, we propose to replace the parameter projection law by a $\sigma$-modification strategy [2]. In this case asymptotic stability can be no longer ensured, but the closed loop system is proven to be globally uniformly ultimately bounded when $L_{\infty}$ disturbances persistently act on the plant dynamics, and a ultimate bound is also computed by using Lyapunov techniques. In the paper we denoted this algorithm as $\sigma$-MCSI (MCS with Integral control action and $\sigma$-modification).

We remark that the closed-loop stability shown here for the proposed adaptive algorithms allows also to prove systematically the boundedness of the integral action of the MCSI and of its associated adaptive gains. Furthermore, even though the main results are initially proven in detail for LTI systems, we show that they can be extended to time-varying linear systems when the dynamics of the plant parameters are slower than those of the adaptive gains [10,11].

The proposed controllers are numerically validated by using two representative sets of examples in which the classical MCS and MCSI algorithms show unbounded adaptive gains. Firstly, we illustrate the main features of the algorithms by considering a continuous-time plant with parameter uncertainty and affected by time-varying disturbances. These disturbances are chosen in accordance with the algorithm to be tested, i.e., they are square integrable for the MCSIPP case, and bounded for the $\sigma$-MCSI algorithm. In the latter case, we show that 
the adaptive gains of the original MCS [10, 11] grow unbounded. Moreover, for the $\sigma$-MCSI we study the closed-loop system response, both in terms of residual tracking error and adaptive gains, when the disturbance is a sinusoidal function with different amplitudes and frequencies. For all cases, boundedness of the closed-loop state is achived as predicted by the theoretical analysis. In the second part, we consider instead an engineering case study, namely, the control of a fullbridge buck DC-AC power converter. The complexity here is due to unmodelled dynamics induced by a discretization of the adaptive laws, the Pulse Width Modulation (PWM) based actuator, and a nonlinear load acting as a disturbance. The tracking performances of the proposed algorithms are compared to those provided by pre-existing MCS solutions whose adaptive gains become unbounded also in this relevant case after a long, but realistic, time interval of interest for the control.

The paper is outlined as follows. The adjusted MCSI adaptive algorithms are introduced in Section 2, while the main theoretical results established therein are proved in Section 3. Simulation results using a numerical example and an engineering-based case study are presented in Sections 4 and 5, respectively. Finally, conclusions are drawn in Section 6.

\section{MCSI algorithms with adjusted adaptive mechanisms}

Consider a plant of the form

$$
\dot{x}=A x+B u+B_{e} \delta, \quad x\left(t_{0}\right) \in \mathbb{R}^{n},
$$

where $x \in \mathbb{R}^{n}$ and $u \in \mathbb{R}$ are the state and the input of the system, respectively, with $n \in \mathbb{N}$ and $t_{0} \in \mathbb{R}$ being the dimension of the state space and the initial time instant, respectively. The scalar disturbance $\delta=\delta(t)$ acting on the plant dynamics belongs either to $L_{2} \cap L_{\infty}$ or $\mathcal{C} \cap L_{\infty}$, with $\mathcal{C}$ being the set of continuous scalar functions. In addition, the system matrices $A \in \mathbb{R}^{n \times n}, B \in \mathbb{R}^{n}$ are in control 
canonical form, i.e.

$$
\begin{aligned}
A & =\left[\begin{array}{ccccc}
0 & 1 & 0 & \ldots & 0 \\
0 & 0 & 1 & \ldots & 0 \\
\vdots & \vdots & \vdots & \ddots & \vdots \\
0 & 0 & 0 & \ldots & 1 \\
a_{1} & a_{2} & \ldots & \ldots & a_{n}
\end{array}\right], \\
B & =\left[\begin{array}{lllll}
0 & 0 & \ldots & 0 & b
\end{array}\right]^{T}=b B_{e} \\
B_{e} & =\left[\begin{array}{lllll}
0 & 0 & \ldots & 0 & 1
\end{array}\right]^{T} .
\end{aligned}
$$

The plant parameters in (2a) and (2b) are assumed to be constant and unknown, but belonging to known bounded sets, i.e., $a_{i} \in\left[a_{i}^{l}, a_{i}^{u}\right]$, where $a_{i}^{u}>a_{i}^{l}, i=1, \ldots, n$, and $b \in\left[b^{l}, b^{u}\right]$, where $b^{u}>b^{l}>0$, with known upper and lower bounds.

This is not as restrictive as it might appear at first. Indeed, nominal values for the plant parameters, as well as their range of variation, are often available in engineering problems. In addition, there is no loss of generality in assuming $b^{u}>b^{l}>0$ because if $0>b^{l}>b^{u}$ then the sign of the adaptive gain weights defined further on should be accordingly selected. Hence, the requirement is, in fact, that $b$ has definite sign.

The control objective for the MCSI algorithm is to impose to system (1) the dynamics of a given reference model while keeping all the closed-loop signals bounded.

More in detail, the reference model is an asymptotically stable LTI system of the form

$$
\dot{x}_{m}=A_{m} x_{m}+B_{m} r, \quad x_{m}\left(t_{0}\right) \in \mathbb{R}^{n},
$$

where $x_{m} \in \mathbb{R}^{n}$ and $r=r(t) \in \mathbb{R}$ are the reference model state and a bounded reference input, respectively, and $A_{m} \in \mathbb{R}^{n}, B_{m} \in \mathbb{R}^{n}$ are the reference model matrices given in the same canonical form as those of the plant, i.e.

$$
\begin{aligned}
A_{m} & =\left[\begin{array}{ccccc}
0 & 1 & 0 & \ldots & 0 \\
0 & 0 & 1 & \ldots & 0 \\
\vdots & \vdots & \vdots & \ddots & \vdots \\
0 & 0 & 0 & \ldots & 1 \\
a_{m, 1} & a_{m, 2} & \ldots & \ldots & a_{m, n}
\end{array}\right], \\
B_{m} & =\left[\begin{array}{lllll}
0 & 0 & \ldots & 0 & b_{m}
\end{array}\right]^{T}=b_{m} B_{e}
\end{aligned}
$$


with $A_{m}$ Hurwitz.

The control action provided by both the MCSI-PP and the $\sigma$-MCSI control algorithms can be written as

$$
u(t)=u_{M C S}(t)+u_{I}(t)
$$

with

$$
\begin{aligned}
u_{M C S}(t) & =K_{X}(t) x(t)+K_{R}(t) r(t), \\
u_{I}(t) & =K_{I}(t) x_{I}(t),
\end{aligned}
$$

where

$$
\dot{x}_{I}=x_{e}+f_{e} \quad \text { and } \quad x_{e}=x_{m}-x ;
$$

the adaptive gains are computed as

$$
\begin{aligned}
K_{X} & =\phi_{X}^{T}+\beta_{X} y_{e} x^{T} \text { and } \dot{\phi}_{X}^{T}=\alpha_{X} y_{e} x^{T}+f_{X}^{T}, \\
K_{R} & =\phi_{R}+\beta_{R} y_{e} r \text { and } \dot{\phi}_{R}=\alpha_{R} y_{e} r+f_{R}, \\
K_{I} & =\phi_{I}^{T}+\beta_{I} y_{e} x_{I}^{T} \text { and } \dot{\phi}_{I}^{T}=\alpha_{I} y_{e} x_{I}^{T}+f_{I}^{T},
\end{aligned}
$$

$\alpha_{X}, \alpha_{R}, \alpha_{I}, \beta_{X}, \beta_{R}$ and $\beta_{I}$ being positive adaptive weights, while

$$
y_{e}=C_{e} x_{e}, \text { with } C_{e}=B_{e}^{T} P, \text { and } P A_{m}+A_{m}^{T} P=-Q, \quad Q=Q^{T}>0 .
$$

Notice that the solution of the Lyapunov equation, $P$, exists because of the Hurwitz assumption for the reference model matrix $A_{m}$.

The functions $f_{e}, f_{X}, f_{R}$ and $f_{I}$, which do not appear in classical MCS algorithms, depend on the strategy exploited to bound the evolution of both the integral variable, $x_{I}$, and the adaptive gains, namely, parameter projection or $\sigma$ modification, and will be described in detail in the following subsections.

We remark that, to derive these terms, the only extra assumption that is required with respect to the MCS algorithms available in the technical literature is that some bounds for the plant parameters are known.

Through the rest of the paper, we denote the diagonal matrices that gather the adaptive gain weights as

$$
\Gamma_{\alpha}=\operatorname{diag}\left(\widehat{\alpha}_{1}, \widehat{\alpha}_{2}, \ldots, \widehat{\alpha}_{2 n+1}\right), \quad \Gamma_{\beta}=\operatorname{diag}\left(\widehat{\beta}_{1}, \widehat{\beta}_{2}, \ldots, \widehat{\beta}_{2 n+1}\right),
$$


with $\left(\widehat{\alpha}_{i}, \widehat{\beta}_{i}\right)=\left(\alpha_{X}, \beta_{X}\right), i=1, \ldots, n,\left(\widehat{\alpha}_{n+1}, \widehat{\beta}_{n+1}\right)=\left(\alpha_{R}, \beta_{R}\right),\left(\widehat{\alpha}_{i}, \widehat{\beta}_{i}\right)=$ $\left(\alpha_{I}, \beta_{I}\right), i=n+2, \ldots, 2 n+1$. In addition, we denote as $\phi$ the stack of the integral part of the adaptive gains, i.e.,

$$
\phi^{T}=\left[\begin{array}{llll}
\phi_{1} & \phi_{2} & \ldots & \phi_{2 n+1}
\end{array}\right]=\left[\begin{array}{lllll}
\phi_{X}^{T} & \vdots & \phi_{R} & \vdots & \phi_{I}^{T}
\end{array}\right] .
$$

Moreover, the vector $\phi^{\star} \in \mathbb{R}^{2 n+1}$ is defined as

$$
\begin{aligned}
\phi^{\star T} & =\left[\begin{array}{llll}
\phi_{1}^{\star} & \phi_{2}^{\star} & \ldots & \phi_{2 n+1}^{\star}
\end{array}\right]=\left[\begin{array}{ccccc}
\phi_{X}^{\star T} & \vdots & \phi_{R}^{\star} & \vdots & \phi_{I}^{\star T}
\end{array}\right] \\
& =\left[\begin{array}{lllll}
b^{-1} B_{e}^{T}\left(A_{m}-A\right) & \vdots & b^{-1} b_{m} & \vdots & \mathcal{O}_{n}^{T}
\end{array}\right],
\end{aligned}
$$

with $\mathcal{O}_{n}$ being the null vector in $\mathbb{R}^{n}$, and $\phi_{X_{j}}^{\star}=b^{-1}\left(a_{m, j}-a_{j}\right), j=1, \ldots, n$. Notice that, as the range of variation of the plant parameters is know, it is possible to find two vectors $\phi^{u}, \phi^{l} \in \mathbb{R}^{2 n+1}$, defined as

$$
\begin{aligned}
\phi^{u T} & =\left[\begin{array}{llll}
\phi_{1}^{u} & \phi_{2}^{u} & \ldots & \phi_{2 n+1}^{u}
\end{array}\right]=\left[\begin{array}{lllll}
\phi_{X}^{u T} & \vdots & \phi_{R}^{u} & \vdots & \phi_{I}^{u T}
\end{array}\right], \\
\phi^{l T} & =\left[\begin{array}{lllll}
\phi_{1}^{l} & \phi_{2}^{l} & \ldots & \phi_{2 n+1}^{l}
\end{array}\right]=\left[\begin{array}{lllll}
\phi_{X}^{l T} & \vdots & \phi_{R}^{l} & \vdots & \phi_{I}^{l T}
\end{array}\right],
\end{aligned}
$$

and a positive constant $\mathcal{M}$ so that

$$
\left\|\phi^{\star}\right\| \leq \mathcal{M}, \quad \phi^{\star} \in \Lambda_{\phi}:=\left[\phi_{1}^{l}, \phi_{1}^{u}\right] \times \cdots \times\left[\phi_{2 n+1}^{l}, \phi_{2 n+1}^{u}\right],
$$

with $\|\cdot\|$ denoting the 2-norm of a vector. Furthermore, let the difference between $\phi^{*}$ and $\phi$ be denoted as

$$
\phi_{e}=\phi^{*}-\phi .
$$

Finally, the elements of the integral adaptive gains evolution $f_{X}, f_{R}$ and $f_{I}$ are collected in a vector defined as:

$$
f^{T}=\left[\begin{array}{llll}
f_{1} & f_{2} & \ldots & f_{2 n+1}
\end{array}\right]=\left[\begin{array}{lllll}
f_{X}^{T} & \vdots & f_{R} & \vdots & f_{I}^{T}
\end{array}\right] .
$$




\subsection{MCSI with parameter projection}

Parameter projection proposes a design for $f$ in (17) that keeps the evolution of $\phi$ within $\Lambda_{\phi}$. This is achieved componentwise as follows:

$$
\begin{aligned}
& f_{X_{j}}(t)=\left\{\begin{array}{c}
0 \text { if } \phi_{X_{j}} \in\left(\phi_{X_{j}}^{l}, \phi_{X_{j}}^{u}\right), \text { or } \phi_{X_{j}}=\phi_{X_{j}}^{l} \text { and } h_{X_{j}} \geq 0, \\
\text { or } \phi_{X_{j}}=\phi_{X_{j}}^{u} \text { and } h_{X_{j}} \leq 0, \\
-h_{X_{j}}(t) \text { otherwise, }
\end{array}\right. \\
& f_{R}(t)=\left\{\begin{array}{c}
0 \text { if } \phi_{R} \in\left(\phi_{R}^{l}, \phi_{R}^{u}\right), \text { or } \phi_{R}=\phi_{R}^{l} \text { and } h_{R} \geq 0, \\
\text { or } \phi_{R}=\phi_{R}^{u} \text { and } h_{R} \leq 0, \\
-h_{R}(t) \text { otherwise, }
\end{array}\right. \\
& f_{I_{j}}(t)=\left\{\begin{array}{c}
0 \text { if } \phi_{I_{j}} \in\left(\phi_{I_{j}}^{l}, \phi_{I_{j}}^{u}\right), \text { or } \phi_{I_{j}}=\phi_{I_{j}}^{l} \text { and } h_{I_{j}} \geq 0, \\
\text { or } \phi_{I_{j}}=\phi_{I_{j}}^{u} \text { and } h_{I_{j}} \leq 0, \\
-h_{I_{j}}(t) \text { otherwise, }
\end{array}\right.
\end{aligned}
$$

with $h_{X_{j}}=\alpha_{X} y_{e} x_{j}, j=1 \ldots n, h_{R}=\alpha_{R} y_{e} r$, and $h_{I_{j}}=\alpha_{I} y_{e} x_{I_{j}}, j=1 \ldots n$.

Notice that, in this way, $f_{j}$ is zero while the corresponding integral gain $\phi_{j}$ is within boundaries, and it is activated when $\phi_{j}$ reaches any of its boundaries with a tendency to leave the region. In this case the parameter projection term freezes $\phi_{j}$ at the corresponding lower or upper admissible interval value.

In turn, a parameter-projection based locking strategy for the integral variable $x_{I}$ is proposed to keep its evolution constrained in a pre-determined region of the phase plane. Let this domain be defined as

$$
\Lambda_{I}:=\left[x_{I_{1}}^{l}, x_{I_{1}}^{u}\right] \times \cdots \times\left[x_{I_{n}}^{l}, x_{I_{n}}^{u}\right],
$$

with $x_{I j}^{l}<0$ and $x_{I j}^{u}>0$, for all $j=1, \ldots, n$. Recalling the definition of $x_{I}$ in (7), its confinement within $\Lambda_{I}$ is achieved through the following scheme:

$$
f_{e_{j}}(t)=\left\{\begin{aligned}
& 0 \text { if } x_{I_{j}} \in\left(x_{I_{j}}^{l}, x_{I_{j}}^{u}\right), \text { or } x_{I_{j}}=x_{I_{j}}^{l} \text { and } x_{e_{j}} \geq 0 \\
& \text { or } x_{I_{j}}=x_{I_{j}}^{u} \text { and } x_{e_{j}} \leq 0 \\
&-x_{e_{j}}(t) \text { otherwise. }
\end{aligned}\right.
$$

Theorem 1. Consider system (1) with $\delta \in L_{2} \cap L_{\infty}$ and the reference model (3). Let the adaptive control action be given by (5)-(9), with the integral part of the adaptive gains in (8) completed with the f-term in (17) computed in accordance with the parameter projection law in (18) and so that $\phi\left(t_{0}\right) \in \Lambda_{\phi}$, 
while the dynamics of $x_{I}$ in (7) are completed with the locking strategy (20) and $x_{I}\left(t_{0}\right) \in \Lambda_{I}$. Then, all the closed-loop signals are bounded and, in particular, $\left(\phi, x_{I}\right) \in \Lambda_{\phi} \times \Lambda_{I}$, and $x_{e} \rightarrow 0$ as $t \rightarrow+\infty$.

Theorem 1 holds for LTV plants as well under the assumption of slow variation of the plant parameters with respect to that of the integral part of the adaptive gains according to the following corollary. It is worth remarking that this is a standard assumption in the MCS literature [11,28].

Corollary 1. Let the dynamic matrix of the plant given in (2) be time-varying, i.e. $A=A(t)$ with $a_{j}=a_{j}(t)$ in the last row of $A(t)$. If, for all $j=1, \ldots, n$,

$$
\frac{\dot{a}_{j}(t)}{b}+\alpha y_{e}(t) x_{j}(t) \approx \alpha y_{e}(t) x_{j}(t),
$$

then Theorem 1 is still verified.

\subsection{MCSI with $\sigma$-modification}

With $\sigma$-modification, the MCSI gains in (8) are computed defining $f$ as:

$$
f_{X}^{T}=-\rho_{X} \sigma_{\phi}(\|\phi\|) \phi_{X}^{T}, \quad f_{R}=-\rho_{R} \sigma_{\phi}(\|\phi\|) \phi_{R}, \quad f_{I}^{T}=-\rho_{I} \sigma_{\phi}(\|\phi\|) \phi_{I}^{T},
$$

where $\rho_{X}, \rho_{R}$, and $\rho_{I}$ are positive constants. The $\sigma$-function is defined as

$$
\sigma_{\phi}(\|\phi\|)=\left\{\begin{array}{l}
0 \text { if }\|\phi\| \leq \widehat{\mathcal{M}}_{\phi}, \\
\eta_{\phi}\left(\frac{\|\phi\|}{\widehat{\mathcal{M}}_{\phi}}-1\right) \quad \text { if } \widehat{\mathcal{M}}_{\phi}<\|\phi\| \leq 2 \widehat{\mathcal{M}}_{\phi}, \\
\eta_{\phi} \text { if }\|\phi\|>2 \widehat{\mathcal{M}}_{\phi},
\end{array}\right.
$$

where

$$
\widehat{\mathcal{M}}_{\phi}=\sqrt{\frac{\lambda_{\max }\left(\Gamma_{\alpha}^{-1} \Gamma_{\rho}\right)}{\lambda_{\min }\left(\Gamma_{\alpha}^{-1} \Gamma_{\rho}\right)}} \mathcal{M},
$$

with $\lambda_{\min }(\mathcal{H})$ and $\lambda_{\max }(\mathcal{H})$ denoting the minimum and maximum eigenvalue of matrix $\mathcal{H}$, respectively, while

$$
\Gamma_{\rho}=\operatorname{diag}\left(\widehat{\rho}_{1}, \widehat{\rho}_{2}, \ldots, \widehat{\rho}_{2 n+1}\right)
$$


with $\widehat{\rho}_{i}=\rho_{X}, i=1, \ldots, n, \widehat{\rho}_{i+1}=\rho_{R}, \widehat{\rho}_{i}=\rho_{I}, i=n+2, \ldots, 2 n+1$, and $\eta_{\phi}$ is a positive constant satisfying

$$
b \eta_{\phi} \lambda_{\min }\left(\Gamma_{\alpha}^{-1} \Gamma_{\rho}\right)>\frac{3}{4} \lambda_{\min }(Q) .
$$

Notice that $\sigma_{\phi}$ is a continuous, non-negative function that becomes activated when the 2-norm of the integral part of the adaptive gains, i.e. $\|\phi\|$, exceeds a certain threshold.

In turn, the locking strategy for $x_{I}$ is now devised in the following $\sigma$-modification fashion

$$
f_{e}^{T}=-\rho_{e} \sigma_{I}\left(\left\|x_{I}\right\|\right) x_{I}^{T}
$$

where $\rho_{e}$ is a positive constant, and $\sigma_{I}(\cdot)$ is defined as

$$
\sigma_{I}\left(\left\|x_{I}\right\|\right)=\left\{\begin{array}{l}
0 \quad \text { if }\left\|x_{I}\right\| \leq \widehat{\mathcal{M}}_{I}, \\
\eta_{I}\left(\frac{\left\|x_{I}\right\|}{\widehat{\mathcal{M}}_{I}}-1\right) \quad \text { if } \widehat{\mathcal{M}}_{I}<\left\|x_{I}\right\| \leq 2 \widehat{\mathcal{M}}_{I}, \\
\eta_{I} \text { if }\left\|x_{I}\right\|>2 \widehat{\mathcal{M}}_{I},
\end{array}\right.
$$

with $\eta_{I}$ and $\widehat{\mathcal{M}}_{I}$ being positive constants.

Let us now define the following vectors and constants

$$
\widetilde{x}_{e}^{T}=\left[\begin{array}{ll}
x_{e}^{T} & \phi_{e}^{T}
\end{array}\right], \quad \widetilde{P}=\left[\begin{array}{cc}
P & \mathcal{O}_{n, 2 n+1} \\
\mathcal{O}_{2 n+1, n} & b \Gamma_{\alpha}^{-1}
\end{array}\right],
$$

where $\mathcal{O}_{\iota, \kappa}$ is the null matrix in $\mathbb{R}^{\iota \times \kappa}$, and

$$
\begin{aligned}
\mu_{1} & =\frac{3}{4} \lambda_{\min }(Q), \\
\mu_{2} & =\frac{3\left\|B_{e}^{T} P\right\|^{2} \Delta_{\infty}^{2}}{\mu_{1}}+\mu_{1}\left(2 \widehat{\mathcal{M}}_{\phi}+\left\|\phi^{\star}\right\|\right)^{2}, \\
\mu & =\sqrt{\frac{\mu_{2}}{\mu_{1}(1-\theta)}}, \quad \theta \in(0,1),
\end{aligned}
$$

with $\Delta_{\infty}$ denoting the $L_{\infty}$ norm of $\delta(t)$.

Theorem 2. Consider system (1) with $\delta \in \mathcal{C} \cap L_{\infty}$ and the reference model (3). Let the adaptive control action be given by (5)-(9), with the integral part of the adaptive gains in (8) completed with the f-term in (17) computed in accordance with the $\sigma$-modification strategy (22), while the dynamics of $x_{I}$ in (7) are completed 
with the locking strategy (27). Then, all the closed-loop signals are bounded and, in particular, $\widetilde{x}_{e}$ is globally uniformly ultimately bounded, i.e., there exists $T$ (dependent on $\widetilde{x}_{e}\left(t_{0}\right)$ and $\mu$ ) and a $\mathcal{K} \mathcal{L}$-class function $\xi: \mathbb{R}^{+} \times \mathbb{R}^{+} \rightarrow \mathbb{R}^{+}$such that

$$
\begin{aligned}
\left\|\widetilde{x}_{e}(t)\right\| & \leq \xi\left(\left\|\widetilde{x}_{e}\left(t_{0}\right)\right\|, t-t_{0}\right), \quad \forall t \in\left[t_{0}, t_{0}+T\right) \\
\left\|\widetilde{x}_{e}(t)\right\| & \leq \sqrt{\frac{\lambda_{\max }(\widetilde{P})}{\lambda_{\min }(\widetilde{P})}} \mu, \quad \forall t \in\left[t_{0}+T,+\infty\right) .
\end{aligned}
$$

Remark 1. Notice that the ultimate bound depends on the magnitude of the disturbance, parameter uncertainty, and design parameters. Moreover, it allows to obtain an upper bound of both the steady-state tracking error and adaptive gains. Indeed, it follows from (29), (15) and (16) that

$$
\left\|x_{e}\right\| \leq\left\|\widetilde{x}_{e}\right\|, \quad\|\phi\| \leq\left\|\widetilde{x}_{e}\right\|+\widehat{\mathcal{M}}_{\phi} .
$$

In addition, it also follows from (29) and (10) that

$$
\begin{aligned}
& \lambda_{\max }(\widetilde{P}) \leq \max \left\{\lambda_{\max }(P), \max _{i=1, \ldots, 2 n+1}\left\{\widehat{\alpha}_{i}\right\} b^{u}\right\}, \\
& \lambda_{\min }(\widetilde{P}) \geq \min \left\{\lambda_{\min }(P), \min _{i=1, \ldots, 2 n+1}\left\{\widehat{\alpha}_{i}\right\} b^{l}\right\} .
\end{aligned}
$$

Finally, according to (12), (15), and (24) one can select

$$
\begin{aligned}
\mathcal{M} & =\frac{1}{b^{l}} \max \left\{\max _{i=1, \ldots, n}\left\{\left|a_{m, i}-a_{i}^{l}\right|,\left|a_{m, i}-a_{i}^{u}\right|\right\},\left|b_{m}\right|\right\}, \\
\widehat{\mathcal{M}}_{\phi} & =\sqrt{\max _{i=1, \ldots, 2 n+1}\left\{\frac{\widehat{\rho}_{i}}{\widehat{\alpha}_{i}}\right\}\left(\min _{i=1, \ldots, 2 n+1}\left\{\frac{\widehat{\rho}_{i}}{\widehat{\alpha}_{i}}\right\}\right)^{-1}} \mathcal{M} .
\end{aligned}
$$

As in the parameter projection case, Theorem 2 is also verified for LTV plants under the assumption made in Corollary 1:

Corollary 2. Let the dynamic matrix of the plant given in (2) be time-varying, i.e. 
$A=A(t)$ with $a_{j}=a_{j}(t)$ in the last row of $A(t)$. If, for all $j=1, \ldots, n$,

$$
\frac{\dot{a}_{j}(t)}{b}+\alpha y_{e}(t) x_{j}(t) \approx \alpha y_{e}(t) x_{j}(t),
$$

then Theorem 2 is still verified.

\section{Proof of the main results}

The stability analysis of the closed-loop system when the MCSI embeds either parameter projection (i.e., MCSI-PP) or $\sigma$-modification terms (i.e., $\sigma$-MCSI) is carried out via Lyapunov theory. Hence, in the following we first derive for the closed-loop dynamics a quadratic auxiliary, Lyapunov-like function that will be subsequently used to prove both Theorem 1, in Section 3.2, and Theorem 2, in Section 3.3.

\subsection{Closed-loop Dynamics}

The closed-loop system can be written in terms of the tracking error and the integral part of the adaptive gains as:

$$
\begin{aligned}
& \dot{x}_{e}=A_{m} x_{e}+B_{e}\left(b \phi_{e}^{T} w-b y_{e} w^{T} \Gamma_{\beta} w-\delta\right), \\
& \dot{\phi}_{e}=-y_{e} \Gamma_{\alpha} w-f,
\end{aligned}
$$

with

$$
w^{T}=\left[\begin{array}{lll}
x^{T} & r & x_{I}^{T}
\end{array}\right] .
$$

Recalling (29), let us consider the following quadratic auxiliary function:

$$
V\left(\widetilde{x}_{e}\right)=\widetilde{x}_{e}^{T} \widetilde{P} \widetilde{x}_{e}
$$

The derivative of this function along the closed-loop system trajectories (36) is:

$$
\begin{aligned}
\dot{V}= & -x_{e}^{T} Q x_{e}+2 x_{e}^{T} P B_{e}\left(b \phi_{e}^{T} w-b y_{e} w^{T} \Gamma_{\beta} w-\delta\right)+ \\
& +2 b \phi_{e}^{T} \Gamma_{\alpha}^{-1}\left(-y_{e} \Gamma_{\alpha} w-f\right)= \\
= & -x_{e}^{T} Q x_{e}-2 b y_{e}^{2} w^{T} \Gamma_{\beta} w-2 b \phi_{e}^{T} \Gamma_{\alpha}^{-1} f-2 y_{e} \delta .
\end{aligned}
$$

After simple algebraic manipulations, and taking into account that both $b$ and all the non-null matrix elements of $\Gamma_{\beta}$ are positive by hypothesis, the derivative of 
the auxiliary function $V$ can be upper bounded as

$$
\begin{aligned}
\dot{V} \leq & -\frac{3}{4} \lambda_{\min }(Q)\left\|x_{e}\right\|^{2}-\lambda_{\min }(Q)\left[\frac{\left\|x_{e}\right\|}{2}-\frac{2\left\|B_{e}^{T} P\right\||\delta|}{\lambda_{\min }(Q)}\right]^{2}+ \\
& +\frac{4\left\|B_{e}^{T} P\right\|^{2}}{\lambda_{\min }(Q)}|\delta|^{2}-2 b \phi_{e}^{T} \Gamma_{\alpha}^{-1} f \leq \\
\leq & -\frac{3}{4} \lambda_{\min }(Q)\left\|x_{e}\right\|^{2}+\frac{4\left\|B_{e}^{T} P\right\|^{2}}{\lambda_{\min }(Q)}|\delta|^{2}-2 b \phi_{e}^{T} \Gamma_{\alpha}^{-1} f .
\end{aligned}
$$

\subsection{Proof of Theorem 1}

Theorem 1 requires the following lemma. The proof is available in Appendix A.

Lemma 1. If $\phi\left(t_{0}\right) \in \Lambda_{\phi}$, then the parameter projection algorithm (18) guarantees

$$
\phi_{e}^{T} \Gamma_{\alpha}^{-1} f \geq 0, \quad \forall t \geq 0
$$

Hence, recalling that $b>0$, (40) becomes

$$
\dot{V} \leq-\frac{3}{4} \lambda_{\min }(Q)\left\|x_{e}\right\|^{2}+\frac{4\left\|B_{e}^{T} P\right\|^{2}}{\lambda_{\min }(Q)}|\delta|^{2} .
$$

Integrating both sides and after some algebraic manipulations, we have

$$
\begin{aligned}
V\left(\widetilde{x}_{e}(t)\right)+\frac{3}{4} \lambda_{\min }(Q) \int_{t_{0}}^{t}\left\|x_{e}(\tau)\right\|^{2} d \tau & \leq V\left(\widetilde{x}_{e}\left(t_{0}\right)\right)+\frac{4\left\|B_{e}^{T} P\right\|^{2}}{\lambda_{\min }(Q)} \int_{t_{0}}^{t}|\delta(\tau)|^{2} d \tau \\
& \leq V\left(\widetilde{x}_{e}\left(t_{0}\right)\right)+\frac{4\left\|B_{e}^{T} P\right\|^{2} \Delta_{2}^{2}}{\lambda_{\min }(Q)}<+\infty
\end{aligned}
$$

with $\Delta_{2}$ denoting the $L_{2}$ norm of $\delta(t)$.

As (43) holds for $t \rightarrow+\infty$ as well, we deduce that $x_{e} \in L_{2}$ and $V\left(\widetilde{x}_{e}\right)$ is bounded. These imply that $x_{e} \in L_{2} \cap L_{\infty}$ and, consequently, $y_{e} \in L_{2} \cap L_{\infty}$. As $r \in$ $L_{\infty}$ and $A_{m}$ is Hurwitz by hypothesis we have that $x_{m} \in L_{\infty}$, this guaranteeing that $x \in L_{\infty}$, while $x_{I} \in \Lambda_{I}$ is ensured by the locking strategy (20) and the fact that $x_{I}(0) \in \Lambda_{I}$ by assumption. In turn, as $\phi\left(t_{0}\right) \in \Lambda_{\phi}$ by hypothesis, the parameter projection algorithm (18) ensures $\phi \in \Lambda_{\phi}$. Therefore, the control gains 
$K_{X}, K_{R}$, and $K_{I}$ are also bounded, which allows to conclude that all the closedloop signals are bounded, including $\dot{x}_{e}$, i.e., $\dot{x}_{e} \in L_{\infty}$.

The boundedness of the closed-loop signals implies that

$$
\frac{d}{d t}\left(\left\|x_{e}\right\|^{2}\right)=2 x_{e}^{T} \dot{x}_{e} \in L_{\infty}
$$

Hence, from Barbalat's Lemma [2] we have that $\left\|x_{e}\right\|^{2} \rightarrow 0$ as $t \rightarrow+\infty$, which implies $x_{e} \rightarrow 0$.

\subsection{Proof of Theorem 2}

Theorem 2 requires the following lemma. The proof is in Appendix B.

Lemma 2. The $\sigma$-modification algorithm (22)-(23) guarantees

$$
\phi_{e}^{T} \Gamma_{\alpha}^{-1} f \geq 0, \quad \forall \phi \in \mathbb{R}^{2 n+1}, \quad \text { and } \quad \phi_{e}^{T} \Gamma_{\alpha}^{-1} f>0, \quad \forall \phi ;\|\phi\| \geq \widehat{\mathcal{M}}_{\phi} .
$$

Moreover,

$$
\phi_{e}^{T} \Gamma_{\alpha}^{-1} f>\frac{\eta_{\phi}}{2} \phi_{e}^{T} \Gamma_{\alpha}^{-1} \Gamma_{\rho} \phi_{e}, \quad \forall\|\phi\|>2 \widehat{\mathcal{M}}_{\phi}
$$

Now, following Theorem 5.1 in [34], we first note that the auxiliary function (38) is continuously differentiable because all the closed-loop signals appearing in $\dot{\widetilde{x}}_{e}$ (see (36)), including $\delta, x_{I}$, and $f$, are continuous. Moreover, $V$ is bounded by two class $\mathcal{K}_{\infty}$ functions $W_{1}\left(\widetilde{x}_{e}\right), W_{2}\left(\widetilde{x}_{e}\right)$ defined as

$$
W_{1}\left(\widetilde{x}_{e}\right) \leq V\left(\widetilde{x}_{e}\right) \leq W_{2}\left(\widetilde{x}_{e}\right),
$$

where $W_{1}\left(\widetilde{x}_{e}\right)=\lambda_{\min }(\widetilde{P})\left\|\widetilde{x}_{e}\right\|^{2}$, and $W_{2}\left(\widetilde{x}_{e}\right)=\lambda_{\max }(\widetilde{P})\left\|\widetilde{x}_{e}\right\|^{2}$.

In what follows we prove that

$$
\dot{V}\left(\widetilde{x}_{e}, t\right) \leq-\widetilde{W}_{3}\left(\widetilde{x}_{e}\right), \quad \text { where } \widetilde{W}_{3}\left(\widetilde{x}_{e}\right):=\mu_{1}\left\|\widetilde{x}_{e}\right\|^{2}-\mu_{2},
$$

with $\mu_{1}, \mu_{2}$ defined in (30).

To show (47), we consider two cases: (i) $\left\|\phi_{e}\right\| \leq 2 \widehat{\mathcal{M}}_{\phi}+\left\|\phi^{\star}\right\|$ and (ii) $\left\|\phi_{e}\right\|>$ $2 \widehat{\mathcal{M}}_{\phi}+\left\|\phi^{\star}\right\|$.

Case (i). When $\left\|\phi_{e}\right\| \leq 2 \widehat{\mathcal{M}}_{\phi}+\left\|\phi^{\star}\right\|$, Lemma 2 and $b>0$ allow to bound $\dot{V}$ 
in $(40)$ as

$$
\begin{aligned}
\dot{V} & \leq-\frac{3}{4} \lambda_{\min }(Q)\left\|x_{e}\right\|^{2}+\frac{4\left\|B_{e}^{T} P\right\|^{2}}{\lambda_{\min }(Q)} \Delta_{\infty}^{2}+\frac{3}{4} \lambda_{\min }(Q)\left(\left(2 \widehat{\mathcal{M}}_{\phi}+\left\|\phi^{\star}\right\|\right)^{2}-\left\|\phi_{e}\right\|^{2}\right)= \\
& =-\left(\mu_{1}\left\|\widetilde{x}_{e}\right\|^{2}-\mu_{2}\right)=-\widetilde{W}_{3}\left(\widetilde{x}_{e}\right) .
\end{aligned}
$$

Case (ii). When $\left\|\phi_{e}\right\|>2 \widehat{\mathcal{M}}_{\phi}+\left\|\phi^{\star}\right\|$, which guarantees $\|\phi\|>2 \widehat{\mathcal{M}}_{\phi}$, Lemma 2, $b>0$ and (26) allow $\dot{V}$ in (40) to be bounded as

$$
\begin{aligned}
\dot{V} \leq & -\frac{3}{4} \lambda_{\min }(Q)\left\|x_{e}\right\|^{2}+\frac{4\left\|B_{e}^{T} P\right\|^{2}}{\lambda_{\min }(Q)}|\delta|^{2}-b \eta_{\phi} \phi_{e}^{T} \Gamma_{\alpha}^{-1} \Gamma_{\rho} \phi_{e} \leq-\frac{3}{4} \lambda_{\min }(Q)\left\|x_{e}\right\|^{2}+ \\
& +\frac{4\left\|B_{e}^{T} P\right\|^{2}}{\lambda_{\min }(Q)} \mid \Delta_{\infty}^{2}-\frac{3}{4} \lambda_{\min }(Q)\left\|\phi_{e}\right\|^{2}+\frac{3}{4} \lambda_{\min }(Q)\left(2 \widehat{\mathcal{M}}_{\phi}+\left\|\phi^{\star}\right\|\right)^{2}= \\
= & -\left(\mu_{1}\left\|\widetilde{x}_{e}\right\|^{2}-\mu_{2}\right)=-\widetilde{W}_{3}\left(\widetilde{x}_{e}\right)
\end{aligned}
$$

Furthermore, defining

$$
W_{3}\left(\widetilde{x}_{e}\right):=\alpha \theta\left\|\widetilde{x}_{e}\right\|^{2}, \quad \theta \in(0,1),
$$

it is immediate that

$$
\widetilde{W}_{3}\left(\widetilde{x}_{e}\right) \geq W_{3}\left(\widetilde{x}_{e}\right), \quad \forall \widetilde{x}_{e} ;\left\|\widetilde{x}_{e}\right\| \geq \mu, \quad \theta \in(0,1),
$$

with $\mu$ defined in (30). Hence, it follows from (48) and (49) that

$$
\dot{V}\left(\widetilde{x}_{e}, t\right) \leq-W_{3}\left(\widetilde{x}_{e}\right), \quad \forall \widetilde{x}_{e} ;\left\|\widetilde{x}_{e}\right\| \geq \mu>0,
$$

with $W_{3}$ being a continuous, positive definite function.

Finally, taking into account that

$$
W_{1}^{-1}\left(W_{2}(\mu)\right)=\sqrt{\frac{\lambda_{\max }(\widetilde{P})}{\lambda_{\min }(\widetilde{P})}} \mu,
$$

the global uniform ultimate boundedness of $\widetilde{x}_{e}$ follows immediately from Theorem 5.1 in [34].

Once the boundedness of both $x_{e}$ and $\phi$ are established, that of the remaining closed-loop signals but $x_{I}$ follow equivalently from the proof of Theorem 1. As 
regards $x_{I}$, its dynamics are

$$
\dot{x}_{I}=-\rho_{e} \sigma_{I}\left(\left\|x_{I}\right\|\right) x_{I}+\psi,
$$

where $\psi=x_{e}$, which has been previously proven to be bounded; hence, $\|\psi(t)\| \leq$ $\Psi$, with $\Psi$ being a positive constant.

By selecting as $V_{I}\left(x_{I}\right)=W_{I 1}\left(x_{I}\right)=W_{I 2}\left(x_{I}\right)=\left\|x_{I}\right\|^{2} / 2$ it is evident that for all $x_{I}, W_{I 1}\left(x_{I}\right) \leq V_{I}\left(x_{I}\right) \leq W_{I 2}\left(x_{I}\right)$. In addition, after a simple algebraic manipulation we have

$$
\begin{aligned}
\dot{V}_{I} & \leq-\rho_{e} \eta_{I}\left(1-\theta_{I}\right)\left\|x_{I}\right\|^{2}-\rho_{e} \eta_{I} \theta_{I}\left\|x_{I}\right\|^{2}+\left\|x_{I}\right\| \Psi \\
& \leq-W_{I 3}\left(x_{I}\right), \quad \text { if } \quad\left\|x_{I}\right\| \geq \mu_{I} \triangleq \max \left\{2 \widehat{M}_{I}, \frac{\Psi}{\rho_{e} \eta_{I} \theta_{I}}\right\}
\end{aligned}
$$

with $W_{I 3}\left(x_{I}\right)=\rho_{e} \eta_{I}\left(1-\theta_{I}\right)\left\|x_{I}\right\|^{2}$ and $\theta_{I}$ being a positive constant chosen in the open interval $(0,1)$.

Consequently, according to Theorem 5.1 in [34], also system (50) is globally uniformly ultimately bounded and there exists some constant $T_{I}$ so that $\left\|x_{I}\right\| \leq \mu_{I}$ for $t \geq T_{I}$. Hence, also the boundedness of $x_{I}$ remains proven.

\subsection{Proof of Corollaries 1 and 2}

When the plant matrix $A$ is constant, then $\phi^{\star}$ defined in (12) is constant as well. Hence, $\dot{\phi}_{e}=-\dot{\phi}$ in (39). When $A=A(t)$ this is no longer true for the first $n$ components of $\phi^{\star}$ :

$$
\dot{\phi}_{e_{j}}=\dot{\phi}_{j}^{*}-\dot{\phi}_{j}=-\frac{\dot{a}_{j}}{b}-\alpha y_{e} x_{j}-f_{j}, \quad j=1, \ldots, n .
$$

However, the approximations in (21) and (35) yield

$$
\dot{\phi}_{e_{j}} \approx \alpha y_{e} x_{j}-f_{j}=-\dot{\phi}_{j}, \quad j=1, \ldots, n .
$$

Consequently, (39) remains invariant and the proofs of Theorems 1 and 2 follow identically.

\section{Numerical validation}

To prove the effectiveness of the MCSI-PP and the $\sigma$-MCSI algorithms presented in Sections 2.1 and 2.2, respectively, here we consider a plant of the form (1) where the matrices in (2) are chosen as 


$$
A=\left[\begin{array}{ccc}
0 & 1 & 0 \\
0 & 0 & 1 \\
-6 & -11 & -6
\end{array}\right], \quad b=6, \quad x(0)=\left[\begin{array}{l}
1.65 \\
1.23 \\
0.94
\end{array}\right] .
$$

The reference model is an LTI system of the form (3) with

$$
A_{m}=\left[\begin{array}{ccc}
0 & 1 & 0 \\
0 & 0 & 1 \\
-120 & -74 & -15
\end{array}\right], \quad b_{m}=240, \quad x_{m}(0)=\left[\begin{array}{c}
-1.4 \\
5.23 \\
-5.06
\end{array}\right]
$$

while the input reference signal is a sinusoidal wave with amplitude 5 and frequency $0.25(\mathrm{rad} / \mathrm{s})$. The disturbance $\delta(t)$ in (1) is either an $L_{2} \cap L_{\infty}$ or a $\mathcal{C} \cap L_{\infty}$ signal, and it is chosen so that the hypothesis required by the gain-locking strategy, i.e., parameter projection or $\sigma$-modification, are satisfied. In both cases, it is activated for the first time at $150 \mathrm{~s}$.

The range of variation assumed for the plant parameters are: $a_{1} \in[-125,50]$, $a_{2} \in[-96,22], a_{3} \in[-43,25], b \in[6,8]$. Moreover, in order to further test the effectiveness of the proposed adaptive algorithms to parameter variation, it is assumed that a sudden change of the plant parameters with respect to their nominal values occur at $\tau=300 \mathrm{~s}$. In particular, after the switch $a_{2}$ becomes 4.4 , while the remaining parameter values undergo a $35 \%$ change. In so doing, the plant to be controlled becomes suddenly unstable.

The adaptive weights are selected as a trade-off between convergence time and reactivity of the control actions. Specifically, we have set $\alpha_{X}=\alpha_{R}=10, \alpha_{I}=1$, $\beta_{X}=\beta_{R}=\alpha_{X} / 10$ and $\beta_{I}=\alpha_{I} / 10$.

\subsection{Results with the MCSI-PP}

According to the possible plant parameter variation and in order to satisfy (15), when the MCSI-PP is adopted we have chosen to limit the integral part of the adaptive gains as follows: $\phi_{X_{1}} \in[-20,1], \phi_{X_{2}} \in[-12,4], \phi_{X_{3}} \in[-5,5]$,

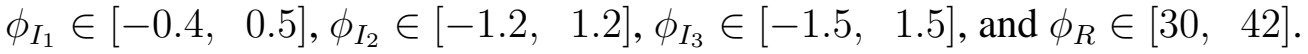

Figure 1 shows the profile of the $L_{2} \cap L_{\infty}$ disturbance, $\delta(t)$, acting on the plant dynamics. In particular it is composed by two pulses with height 125 , width 50 s, and starting at 150 s and 300 s, respectively. Notice that the plant parameters change jointly with the second activation of the disturbance. Hence, in what follows we also study the effect of a double perturbation on the closed loop dynamics occurring at the same time. 


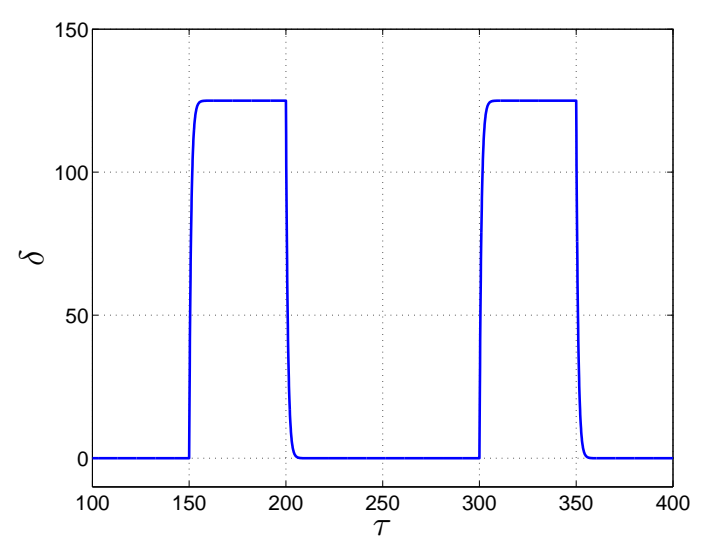

Figure 1: Square-integrable disturbance used in the MCSI-PP case.

Figure 2 shows the effectiveness of the proposed algorithm to impose the model reference dynamics to the plant (1) with uncertain parameters before and after the activation of the disturbance in Figure 1. Precisely, Figure 2a shows that the adaptive strategy makes the closed-loop state trajectory (blue line) converge to the steady-state periodic solution of the reference model (red line) before the activation of the disturbance. This is confirmed by Figure $2 \mathrm{~b}$, which shows the precise matching of each state variable of the plant with the corresponding state variable of the reference model in the steady-state regime also before the activation of the disturbance, and by the corresponding tracking errors converging to zero depicted in Figure 2c. Finally, Figure $2 \mathrm{~d}$ portrays the effect of both the disturbance in Figure 1 and the previously described parametric variations on the tracking error. It is interesting to note that, despite the presence of disturbances and variation of the plant parameters, the tracking error remains bounded and converges to zero after a transient -due to the disturbance activation/deactivation- in accordance with Theorem 1.

The evolution of the integral part of the adaptive gains (8) is reported in Figure 3. As clearly shown, and according to Theorem 1, these gains are always bounded in the preassigned set. In addition, during the activation of the disturbance some adaptive gains are locked, thus preventing a possible drift. Specifically, at the activation of the disturbance in Figure 1, during some time intervals $\phi_{X_{1}}, \phi_{X_{2}}$ (see Figure 3a), $\phi_{R}$ (see Figure 3b), $\phi_{I_{1}}$ (see Figure 3c) are saturated both at their upper and lower bounds, while $\phi_{I_{2}}$ and $\phi_{I_{3}}$ are locked at their lower bound and upper bound, respectively (see Figure 3c). Notice that upper and/or lower gain bounds are indicated in Figure 3 for those adaptive gains which are 


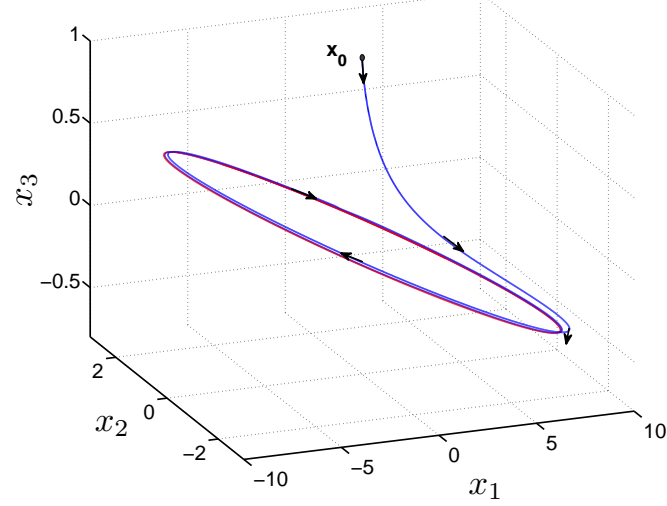

(a)

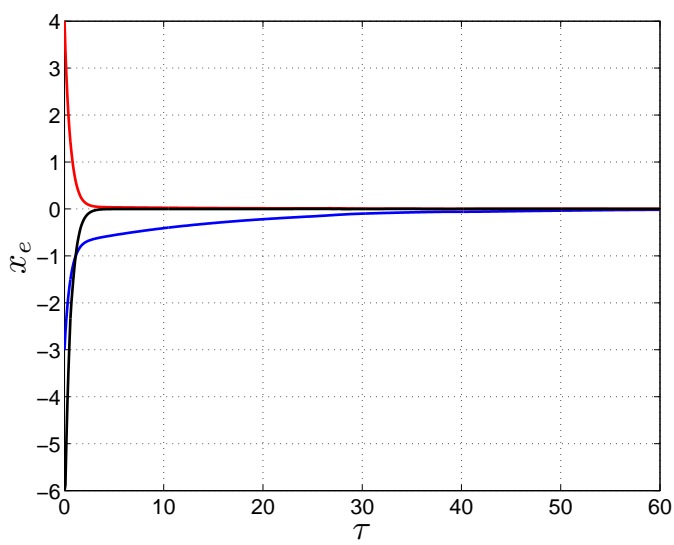

(c)
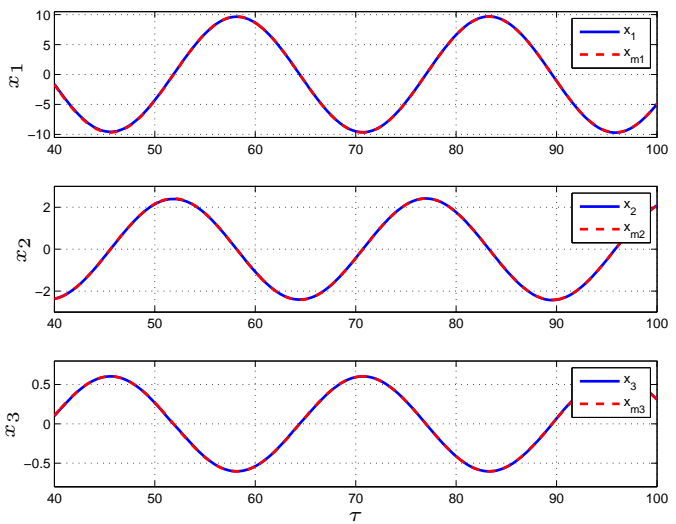

(b)

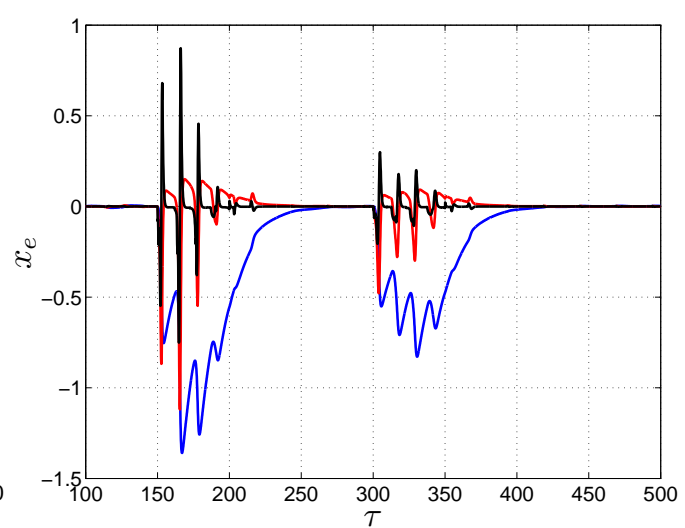

(d)

Figure 2: Tracking performance of the MCSI-PP algorithm. (a) Phase portrait of the plant trajectory (blue line) converging to the steady-state periodic solution of the reference model (red line) before the activation of the disturbance; (b) closedloop plant trajectory (blue solid line) and reference model dynamics (red dashed line) in steady state regime before the activation of the disturbance; tracking error before (c) and after (d) the activation of the disturbance: $x_{e_{1}}$ (blue line), $x_{e_{2}}$ (red line), $x_{e_{3}}$ (black line). 
locked. These limits are depicted as dotted lines with the color of the corresponding adaptive gain. In addition, Figures $2 d$ and 3a-3c clearly indicate that the adaptive gains reenter the non-locking region when the tracking error converges to zero.

Furthermore, as shown in Figure $3 \mathrm{~d}$ the control gain $\phi_{I}$ saturates during the initial transient, but all its components enter into the non-locking region as the tracking error goes to zero. Similarly, also the gain $\phi_{R}$ is locked for some time intervals before the activation of the disturbance, although a zoom of its dynamics are not shown for the sake of brevity.

Finally, we remark that, as the tracking error and the integral part of the adaptive gains are bounded (see Figures 2 and 3, respectively), then the control action in (5), the proportional part of the adaptive gains, and consequently those in (8), remain bounded. Nevertheless, the time evolution of these closed-loop variables is not reported here again for the sake brevity.

\subsection{Results with the $\sigma$-MCSI}

In order to show the effectiveness of the $\sigma$-MCSI to reject persistent disturbances, we analyze the case when the disturbance $\delta$, acting on the plant (1) with matrices in (52), is a signal of the form $\delta(\tau)=8 \cdot 10^{2} \sin (\pi t / 10)$ that becomes active at $\tau=150 \mathrm{~s}$. Notice that the reference model matrices and the reference input signal $r$ are those used in Section 4.1. Furthermore we set $\eta_{\phi}=5 \cdot 10^{-4}$ in (23), $\Gamma_{\rho}$ in (25) is chosen as $\Gamma_{\rho}=\Gamma_{\alpha}$ and $\mathcal{M}=46$ so that (15) holds.

Figure 4 shows the tracking performance over a long control horizon. Specifically, Figure 4a confirms that the tracking error before the disturbance activation is similar to that obtained when the MCSI-PP is used (see Figure 2c). The presence of the sinusoidal disturbance induces a residual tracking error which is shown in Figure 4b. However, this tracking error is small when compared to the amplitude of the reference model variables (see Figure $2 b$ ). Precisely, this residual tracking error of each state variable is more than 50 times smaller than the corresponding component of the reference model vector.

We remark that, despite the disturbance acts persistently on the closedloop dynamics, the integral parts of the adaptive control gains, i.e., $\phi_{X}, \phi_{R}$ and $\phi_{I}$, are kept bounded as clearly shown in Figures 5a, 5 b and 5c, respectively. From the evolution of $\|\phi\|$, depicted in Figure (5d) together with the thresholds $\widehat{\mathcal{M}}_{\phi}$ and $2 \widehat{\mathcal{M}}_{\phi}$ used in the definition of the $\sigma$-modification strategy in (22)-(23), additional insights into closed-loop behavior can be drawn. Indeed, at the activation of the disturbance, this norm rapidly increases and crosses the threshold $2 \widehat{\mathcal{M}}_{\phi}$. Consequently, a constant leakage factor, i.e., $\eta_{\phi}$, is used to bound 


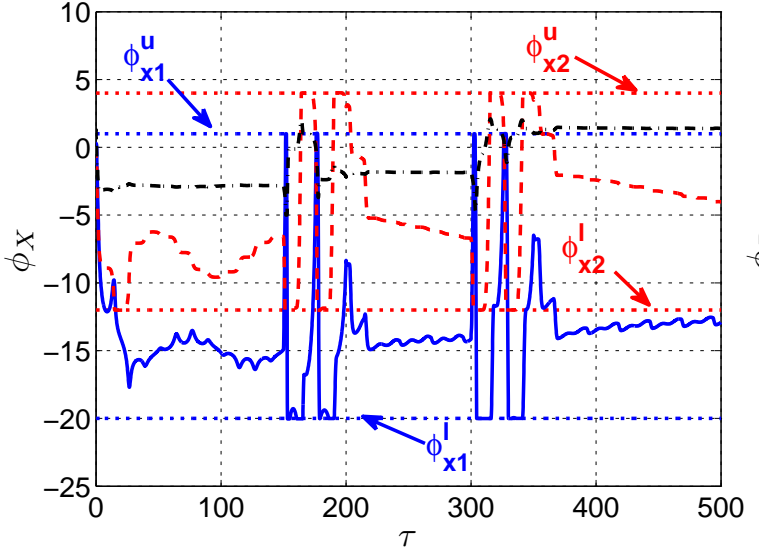

(a)

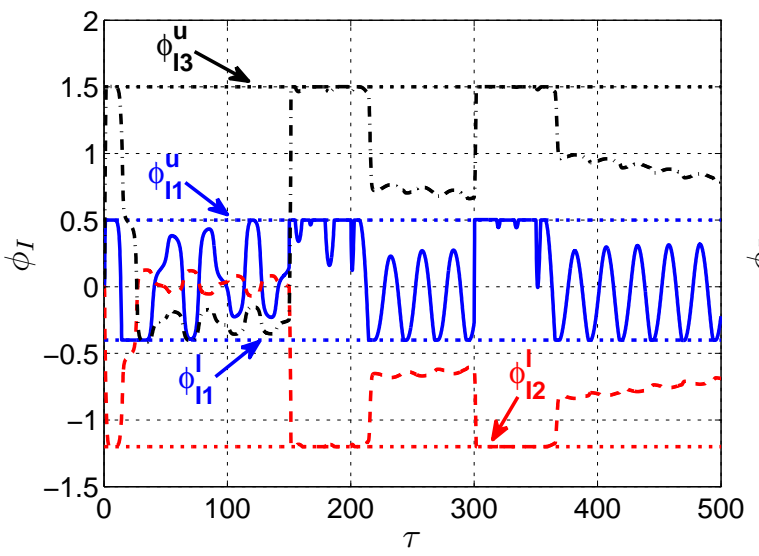

(c)

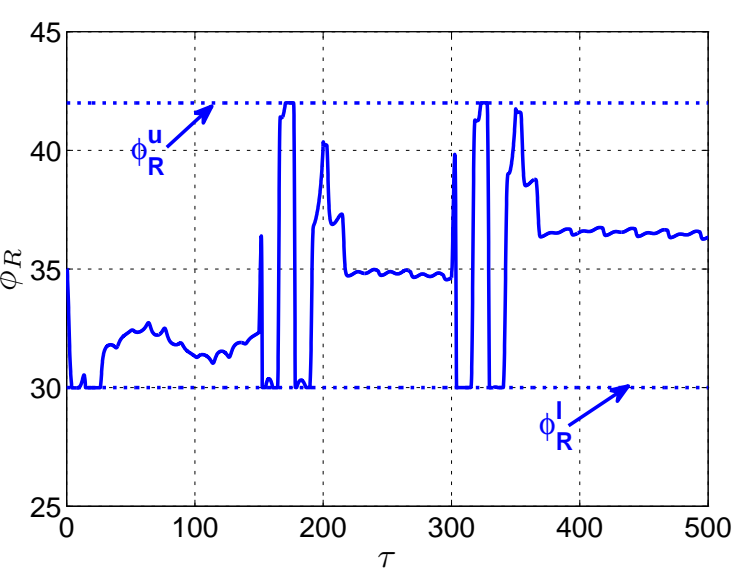

(b)

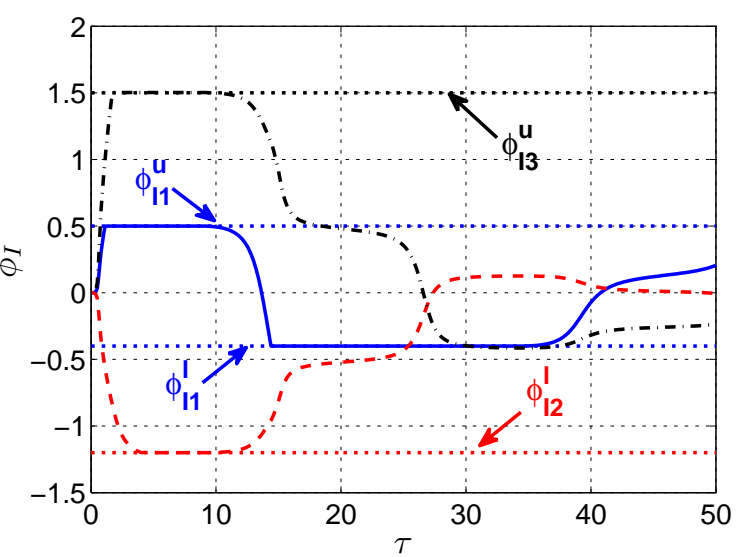

(d)

Figure 3: Integral part of the adaptive gains with the MCSI-PP algorithm. (a) $\phi_{X}$ : $\phi_{X_{1}}$ (blue solid line), $\phi_{X_{2}}$ (red dashed line) and $\phi_{X_{3}}$ (black dashed-dotted line) (b) $\phi_{R}$, (c) $\phi_{I}: \phi_{I_{1}}$ (blue solid line), $\phi_{I_{2}}$ (red dashed line) and $\phi_{I_{3}}$ (black dashed-dotted line) (d) $\phi_{I}$ at the beginning of the simulation. 


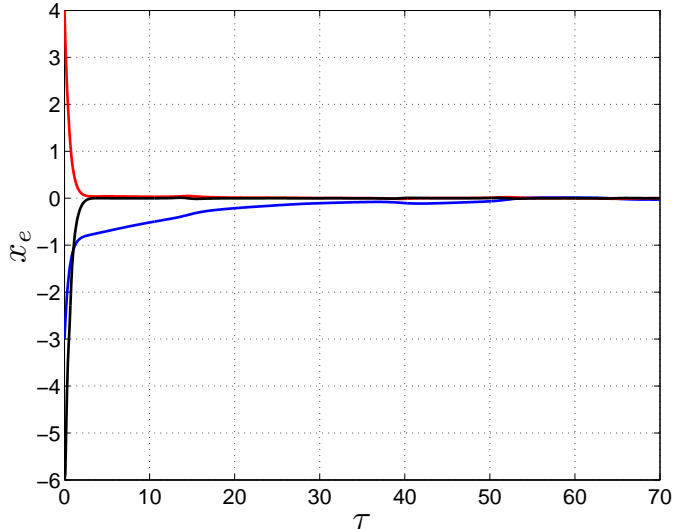

(a)

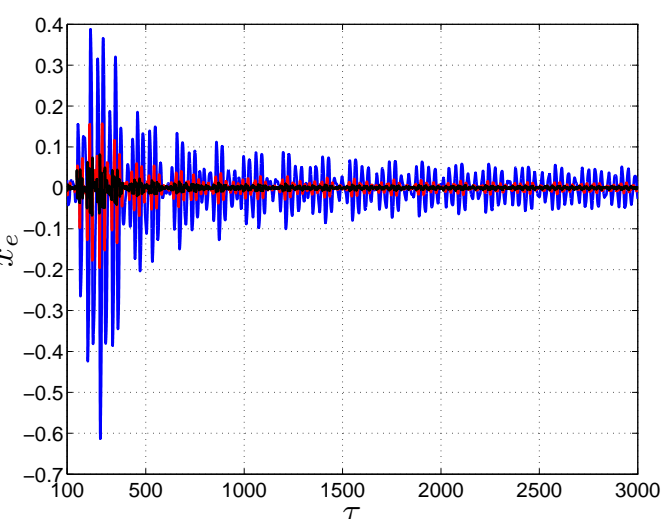

(b)

Figure 4: Tracking performance of the $\sigma$-MCSI algorithm. Tracking error before (b) and after (c) the activation of the disturbance: $x_{e_{1}}$ (blue line), $x_{e_{2}}$ (red line), $x_{e_{3}}$ (black line).

the growth of the integral part of the adaptive gains. After that, we have $\widehat{\mathcal{M}}_{\phi} \leq$ $\|\phi\| \leq 2 \widehat{\mathcal{M}}_{\phi}$, and according to (23) the integral part of the adaptive gains further decreases with a leakage factor which scales on the basis $\|\phi\|$. Finally, when time goes to infinity, these gains enter in the non-locking region $\left(\|\phi\| \leq \widehat{\mathcal{M}}_{\phi}\right)$. At first there is still the need of the additional term (22) in (8) to prevent gain drifting as $\|\phi\|$ periodically crosses the $\widehat{\mathcal{M}}_{\phi}$-value, i.e., $\tau \in[1500,2200] \mathbf{s}$, but at the end the re-entry into the non-locking region is permanent.

Similar to the case reported in Section 4.1, as the integral part of the adaptive gains and the tracking error are bounded then the control action in (5), the proportional part of the adaptive gains, and consequently those in (8), remain bounded. The time evolution of these closed-loop variables is not portrayed here for the sake of brevity.

It is important to point out that the boundedness of the MCSI adaptive gains when time goes to infinity is guaranteed by the $\sigma$-modification strategy. Indeed, under the same working conditions, the adaptive gains of the classical MCS diverge as depicted in Figures $6 \mathrm{a}$ and $6 \mathrm{~b}$. Consequently, the norm of the integral part of the control gains grows unbounded as shown in $6 c$.

We conclude the analysis of the $\sigma$-MCSI by considering the closed-loop response for several disturbances of the form $\delta(\tau)=Q \sin (\omega \tau)$, with $Q \in[100,1500]$, $\omega \in[0.1,1.5]$ and $\tau \in \mathcal{T}=\left[0,3 \cdot 10^{3}\right]$. Figure 7 shows the maximum value of the 


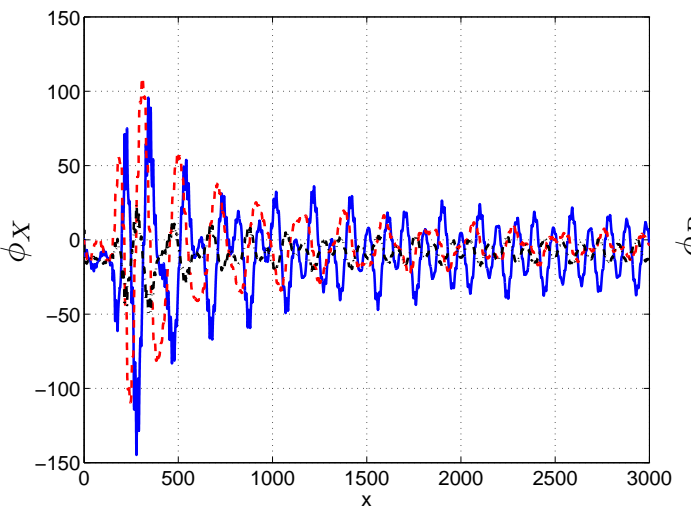

(a)

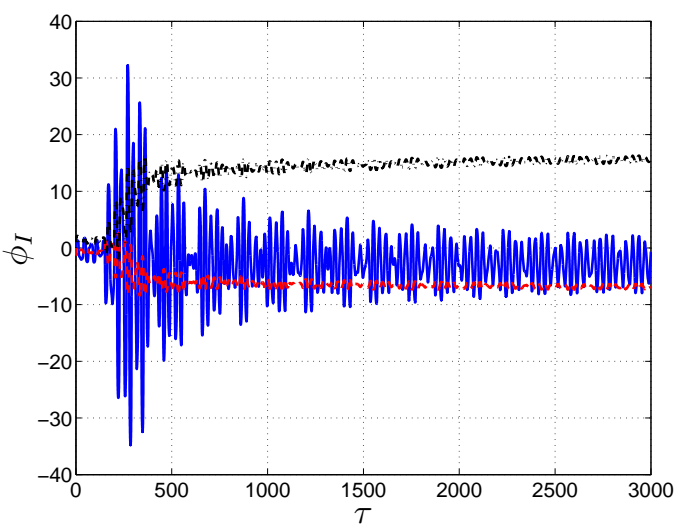

(c)

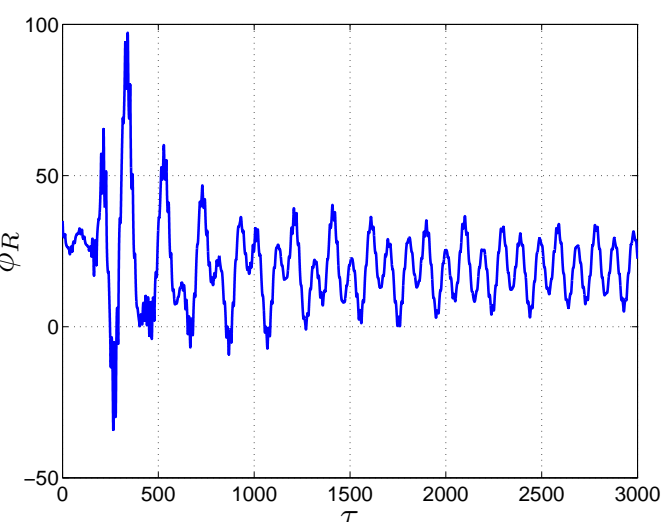

(b)

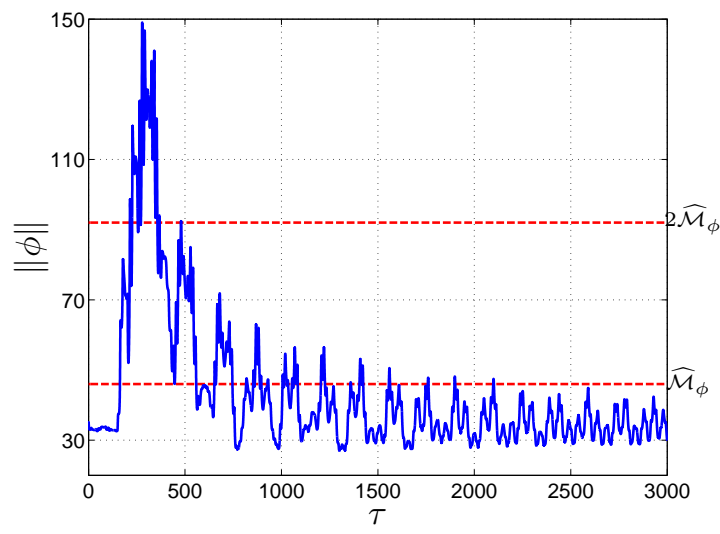

(d)

Figure 5: Integral part of the adaptive gains with the $\sigma$-MCSI algorithm. (a) $\phi_{X}$ : $\phi_{X_{1}}$ (blue solid line), $\phi_{X_{2}}$ (red dashed line) and $\phi_{X_{3}}$ (black dashed-dotted line) (b) $\phi_{R}$, (c) $\phi_{I}: \phi_{I_{1}}$ (blue solid line), $\phi_{I_{2}}$ (red dashed line) and $\phi_{I_{3}}$ (black dashed-dotted line) (d) bounded evolution of $\|\phi\|$. 


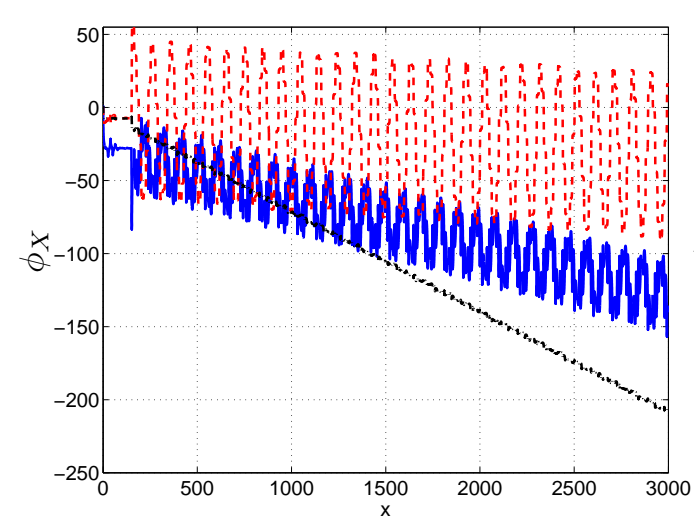

(a)

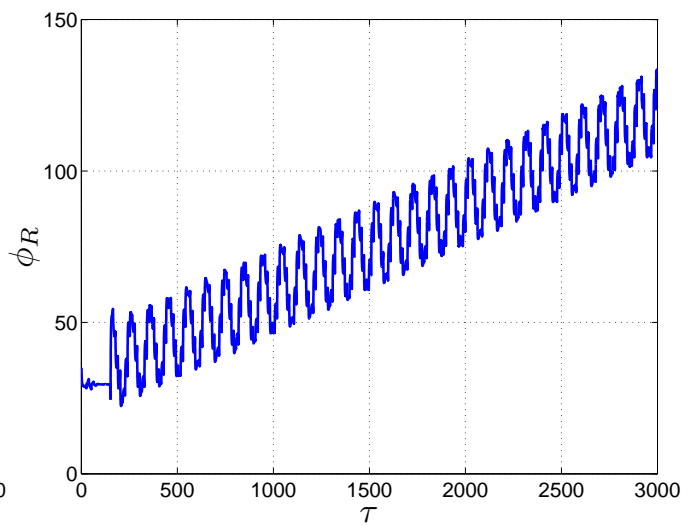

(b)

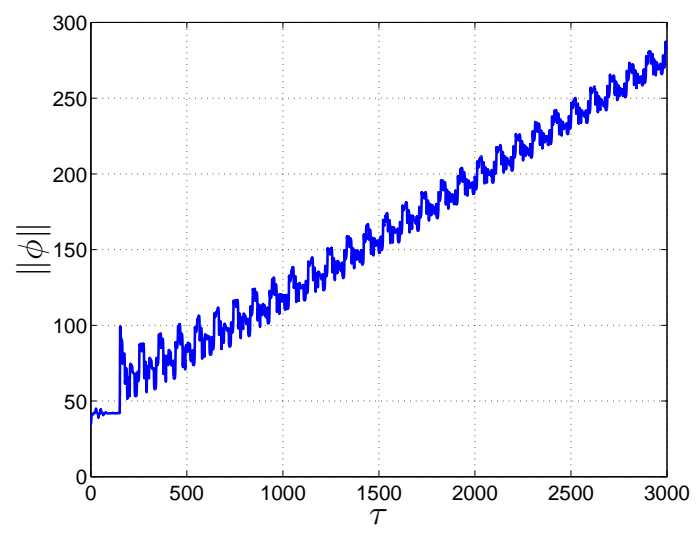

(c)

Figure 6: Evolution of the integral part of the MCS adaptive gains without any locking strategy in the face of a persistent sinusoidal disturbance: (a) $\phi_{X}: \phi_{X_{1}}$ (blue solid line), $\phi_{X_{2}}$ (red dashed line) and $\phi_{X_{3}}$ (black dashed-dotted line) (b) $\phi_{R}$, (c) diverging evolution of $\|\phi\|$. 
norm of the residual error $\left\|x_{e}\right\|$ (Figure 7a) and the maximum value of the norm of the integral part of the adaptive gains $\|\phi\|$ (Figure 7b) computed over $\mathcal{T}$ for each pair $(Q, \omega)$ in steady-state condition. We remark that not only the control strategy provides a bounded tracking error and bounded adaptive gains in any working condition, but also a small residual error. Indeed, the norm of the steady-state tracking error in the worst case (upper right corner in Figure 7a) is more than 30 times smaller than the norm of the reference model vector. This confirms the effectiveness of the approach to limit the growth of the adaptive gains without altering tracking performance.

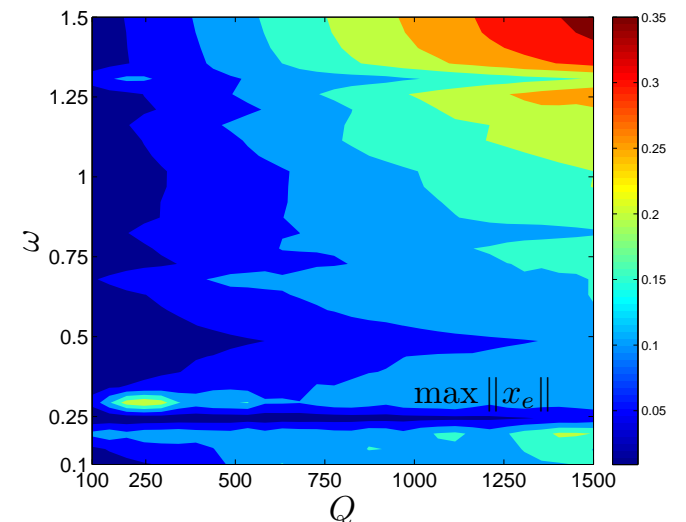

(a)

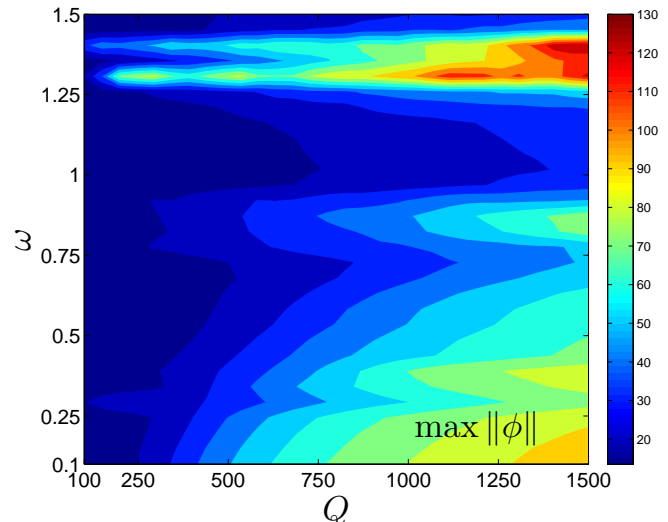

(b)

Figure 7: Tracking performance for different sinusoidal disturbances of the form $Q \sin (\omega \tau)$ : (a) maximum norm of the residual tracking error, $x_{e}$, after transient, and (b) maximum norm of the integral part of the adaptive gains, $\phi$, after transient.

\section{Engineering case study}

To further confirm the effectiveness of the proposed improvements to the preexisting MCS and MCSI algorithms, we consider here the control of a full bridge buck inverter with a nonlinear load, see Figure 8a. The challenge is to impose a periodic behavior to the system response with a limited knowledge of the plant parameters, and in the face of unknown nonlinear disturbances and unmodelled dynamics (e.g., digital implementation of continuous-time control laws) over a long time interval. Indeed, for practical applications, such as uninterruptible power systems, these kind of devices must provide a periodic stable voltage to loads over 
many cycles [35]. In this work we assume that the nonlinear current $i_{N}$ is periodic and takes the shape depicted in Figure 8b, while as physical parameters we select $E=220 \mathrm{~V}, C=40 \mu \mathrm{F}$, and $L=6 \mathrm{mH}$ with a parasitic resistance of $r=0.2 \Omega$.

The control objective is to impose to the output voltage, $v_{C}$, a sinusoidal reference profile $\widehat{v}_{C}(t)=M \sin (2 \pi \nu t)=M \sin (\widehat{\omega} t)$, with $M=E$ and $\nu=50$ $\mathrm{Hz}$.

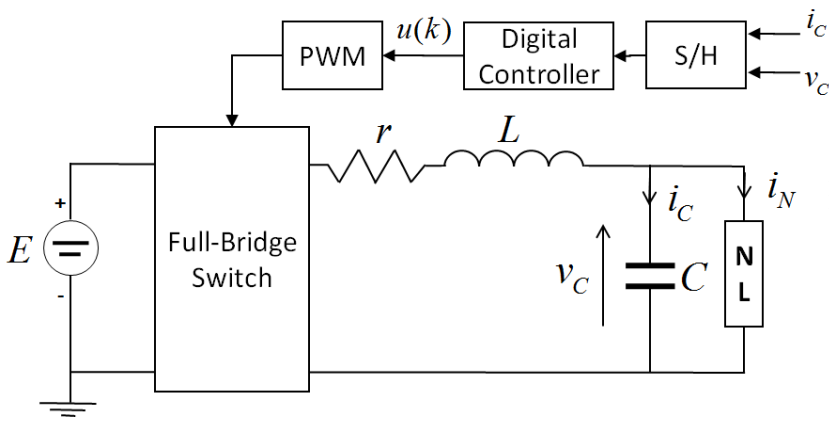

(a)

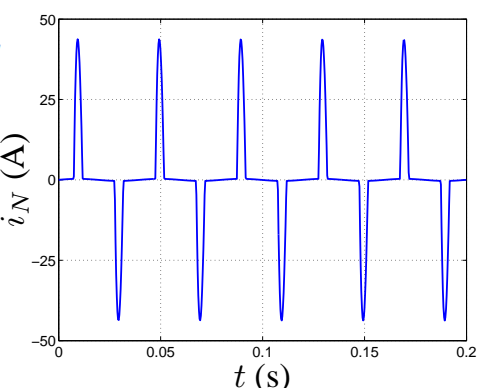

(b)

Figure 8: (a) Schematic of the full bridge buck inverter, and (b) time history of the periodic nonlinear load current that acts as a disturbance.

By defining as state vector $z=\left[\begin{array}{ll}v_{C} & i_{C}\end{array}\right]^{T}$, from Kirchhoff's laws the system dynamics are

$$
\dot{z}=\widehat{A} z+\frac{E}{L C} B u+\frac{1}{L C} B \widehat{d}, \quad \widehat{d}=-\left(r i_{N}+L \frac{d i_{N}}{d t}\right),
$$

where $u \in[-1,+1]$ is the PWM duty cycle, and $\widehat{d}$ is the disturbance due to the nonlinear load, while the system matrices are

$$
\widehat{A}=\left[\begin{array}{cc}
0 & 1 \\
-\frac{1}{L C} & -\frac{r}{L}
\end{array}\right], \quad B=\left[\begin{array}{l}
0 \\
1
\end{array}\right] .
$$

\section{Let us define the reference model}

$$
\dot{z}_{m}=\widehat{A}_{m} z_{m}+B \widehat{r}, \quad \widehat{r}(t)=\frac{2 M q \widehat{\omega}}{\sqrt{L C}} \cos (\widehat{\omega} t), \quad z_{m}\left(t_{0}\right)=\left[\begin{array}{ll}
0 & M \hat{\omega}^{2}
\end{array}\right]^{T},
$$

where $t_{0}$ is the initial time instant, $q$ is a positive constant, and the dynamic 
matrix $\widehat{A}$ is given by

$$
\widehat{A}_{m}=\left[\begin{array}{cc}
0 & 1 \\
-\widehat{\omega}^{2} & -\frac{2 q}{\sqrt{L C}}
\end{array}\right] .
$$

One can easily check, for example by substitution or by resorting to the Laplace domain, that $z_{m_{1}}(t)=M \sin (\widehat{\omega} t)$. Hence, the control aim of imposing the required sinusoidal shape, $\widehat{v}_{C}(t)$, to the voltage across the capacitor $C$ can be reformulated as a model following problem of the state, $z_{m}$, of the reference model (56), by the state, $z$, of the plant (54).

The entries of the matrices in (54) and (57) can be very large. Hence, for control purposes and in order to avoid numerical problems it is advisable to make them dimensionless. This can be achieved with the following change of coordinates and time scaling

$$
x_{1}=\frac{z_{1}}{E}, \quad x_{2}=\frac{\sqrt{L C}}{E} z_{2}, \quad \tau=\frac{t}{\sqrt{L C}} .
$$

Notice that the transformation which maps $z$ onto $x$ is linear and therefore it can be also expressed as $x=\mathcal{W} z$, where $\mathcal{W}$ is defined as

$$
\mathcal{W}=\frac{1}{E}\left[\begin{array}{cc}
1 & 0 \\
0 & \sqrt{L C}
\end{array}\right]
$$

After some algebraic manipulations it is possible to show that, in the new variables, the plant dynamics answers to:

$$
\frac{d x}{d \tau}=A x+B u+B d(\tau)
$$

with

$$
A=\left[\begin{array}{cc}
0 & 1 \\
-1 & -r \sqrt{\frac{L}{C}}
\end{array}\right], \quad \text { and } \quad d(\tau)=\frac{1}{E}\left(r i_{N}(\tau)+\sqrt{\frac{L}{C}} \frac{d i_{N}}{d \tau}(\tau)\right) .
$$

In turn, the reference model dynamics are

$$
\frac{d x_{m}}{d \tau}=A_{m} x_{m}+B r(\tau),
$$




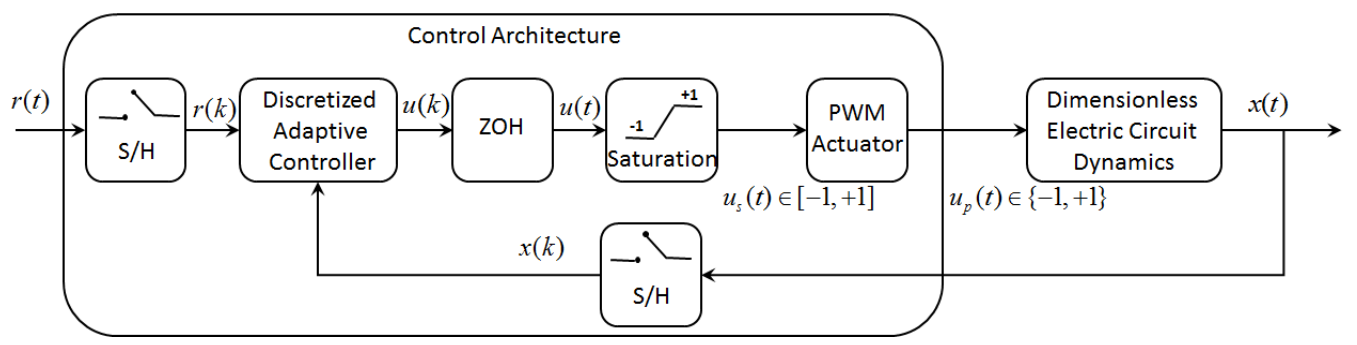

Figure 9: Control scheme.

with

$$
A_{m}=\left[\begin{array}{cc}
0 & 1 \\
-\omega_{0}^{2} & -2 q
\end{array}\right], \quad \text { and } \quad r(\tau)=2 q \omega_{0} \cos \left(\omega_{0} \tau\right)
$$

where $x_{m}=\mathcal{W} z_{m}$, with $\mathcal{W}$ being the matrix in (59), and $\omega_{0}=\widehat{\omega} \sqrt{L C}$.

In the following subsection we test the tracking performance of a set of MCS algorithms. Specifically, we consider the MCS presented in [36], the MCSI without any locking method introduced in [37], and finally the MCSI-PP and the $\sigma$ MCSI presented in Section 2. These controllers have been discretized by means of the Tustin discretization method and used within the control scheme depicted in Figure 9. Notice that this control architecture includes the PWM actuator, a saturation, sample and hold $(\mathrm{S} / \mathrm{H})$ and zero-order hold $(\mathrm{ZOH})$ blocks, which are commonly used to operate power converters. Hence, we test robustness not only with respect to discretization, but also with respect to additional nonlinearities that have not been taken into account during the design of the controllers, i.e., additional unmodelled nonlinear dynamics. A discrete time adaptive controller without locking strategies for a full bridge buck inverter operating with a linear load has been presented in [38].

Taking into account the frequency response of system (54), the PWM frequency has been set to $20 \mathrm{kHz}$, while the controllers have been discretized with a sampling frequency of $15 \omega_{T}$, with $\omega_{T}$ being the cut-off frequency of the circuit.

All the adaptive strategies have been tuned with the same adaptive weights, which have been chosen again as a trade-off between convergence time and reactivity of the control actions. Precisely, we have set $\alpha_{X}=10^{-2}, \alpha_{R}=10^{-3}$, $\alpha_{I}=5 \cdot 10^{-3}, \beta_{X}=\alpha_{X} / 10, \beta_{R}=\alpha_{R} / 10$ and $\beta_{I}=\alpha_{I} / 10$, and the locking strategies in Section 2 have been tuned so that (15) is fulfilled. In addition, in the case of $\sigma$-modification, we have set $\Gamma_{\rho}=\operatorname{diag}(0.01,0.01,0.01,0.5,0.5)$.

Finally, with the aim of investigating the evolution of the adaptive gains over a long manoeuvre, the time interval of interest for the control has been set to 
$\mathcal{T}=\left[0,2 \cdot 10^{4}\right]$

\subsection{Numerical analysis}

For the working conditions described above, the control gains of the MCS solutions available in the literature diverge over time due to the presence of the disturbance caused by the nonlinear load. This is shown in Figure 10a, which depicts the norm of the adaptive gains of both the MCS strategy (black line) and the pre-existing MCSI algorithm (green line) over the entire control horizon. Specifically, Figure 10b reveals that the MCS adaptive gains diverge from $\tau>10^{3}$, while in the case of the pure MCSI strategy there is at first a slow gain drifting till $\tau=9 \cdot 10^{3}$, but then the adaptive gains increase unbounded. This unwanted phenomena is suppressed when either the MCSI-PP (red line) or the $\sigma$ MCSI (blue line) are used, and the adaptive gains remain bounded over the entire manoeuvre in accordance with the theoretical predictions.

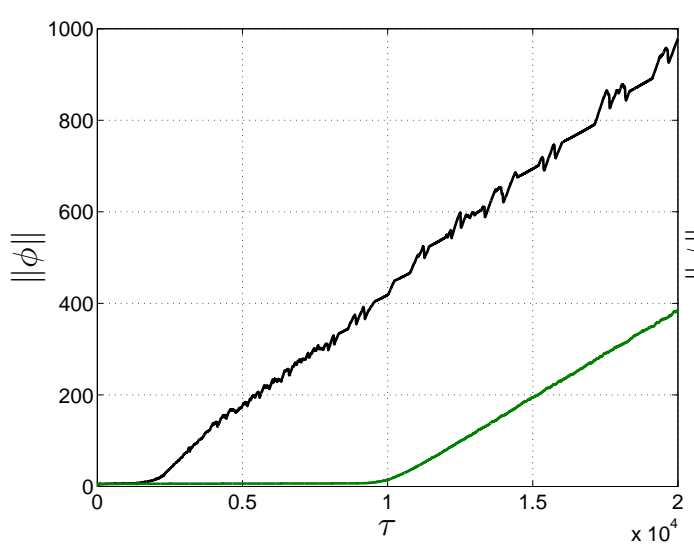

(a)

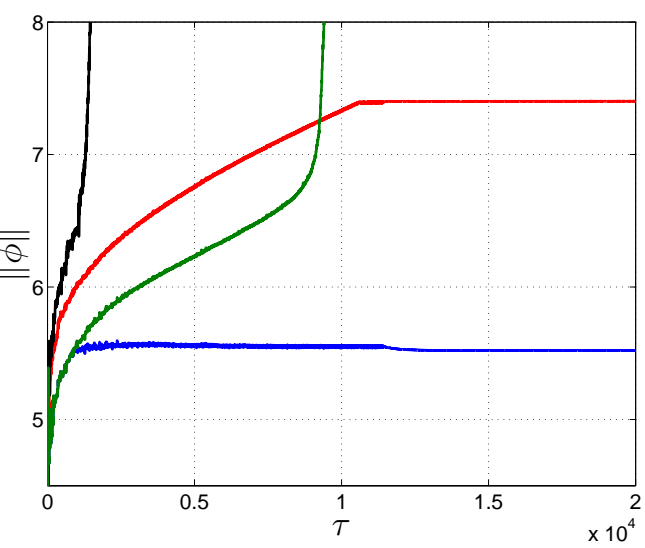

(b)

Figure 10: Evolution of $\|\phi\|$ for different MCS strategies. (a) MCS (black line) and MCSI (green line); (b) MCS (black line), MCSI (green line), MCSI-PP (red line) and $\sigma$-MCS (blue line).

We point out that the divergence of the adaptive gains alters closed-loop tracking performance. In particular, in the case of the MCS strategy it is possible to observe in Figure 11a a loss of tracking performance during the onset of the control gains instability around $\tau=10^{3}$. Here, as depicted in Figure $11 \mathrm{~b}$, the MCS tracking error (black line) increases from less than a $2 \%$ up to a $15 \%$, which is not admissible for this specific engineering application. Furthermore, the tracking 
error keeps growing with time till tracking is completely lost around $\tau=2.3 \cdot 10^{3}$, as apparent in Figures 11c and 11d.

A similar phenomena also occurs when the MCSI algorithm is exploited to tackle the dynamics of the buck inverter, see Figure 12. Even though in this case the classical MCSI tracking error does not exceed 30\% (see green line in Figure 12d), such a value is not acceptable in the context of power electronic engineering as alternative solutions in the technical literature provide better closed-loop responses, see for example [39]. Instead the MCSI-PP and $\sigma$-MCSI provide low tracking error over the entire manoeuvre.

We remark that both gain divergence and loss of tracking performance are the result of unmodeled dynamics due to control discretization and hard nonlinearities, e.g., saturations [40].

Figures 11 and 12 also illustrate the excellent and robust closed-loop tracking performance obtained when both the MCSI-PP (red line) and the $\sigma$-MCSI (blue line) are inserted in the control loop displayed in Figure 9. To better appreciate this crucial feature, the residual tracking errors provided by these control algorithms are also depicted in Figure 13: both of them are very small when compared to the amplitude of the reference signal, which is unitary.

Finally, we point out that, although the control action applied to the plant cannot diverge because of the saturation block in the control architecture in Figure 9, the resulting input of the PWM actuator can be highly nonsmooth when gain instability is induced. This additional unwanted effect is clear in Figure 14 for the MCS (black line) and MCSI (green line) algorithms. Instead, smooth control actions that are always within the possible range of variation (i.e., $[-1,1]$ ), are provided by the MCSI-PP (red line) and the $\sigma$-MCSI (blue line) strategies.

\section{Conclusions}

Model reference adaptive controllers belonging to the class of minimal control synthesis algorithms are an effective solution to steer the dynamics of plants with uncertain parameters and affected by unmodelled nonlinear dynamics and disturbances. Nonetheless, such disturbances can induce an unbounded growth of the adaptive gains that eventually jeopardizes both tracking performance and stability. In this paper we have proposed continuous-time MCSI algorithms which include either parameter projection or $\sigma$-modification strategies to prevent the onset of such undesirable dynamics.

The former allows to preserve convergence to zero of the tracking error with respect to bounded $L_{2}$-disturbances while keeping the adaptive gains within a 


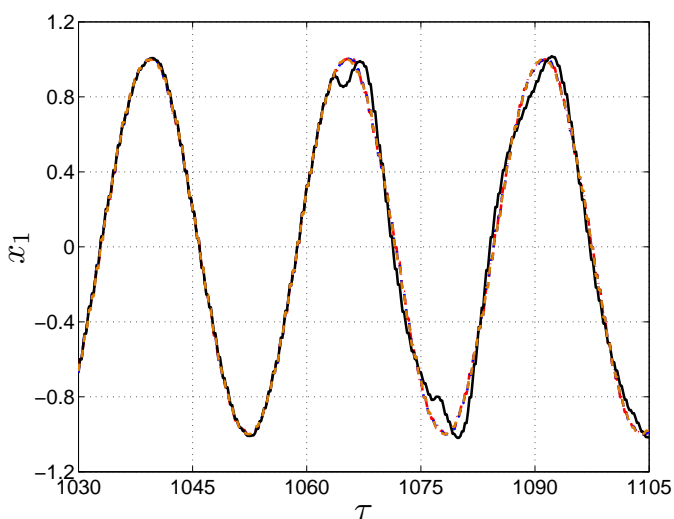

(a)

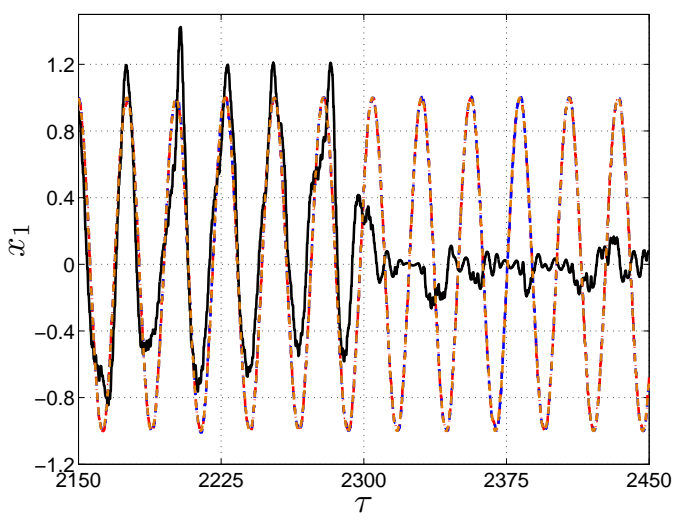

(c)

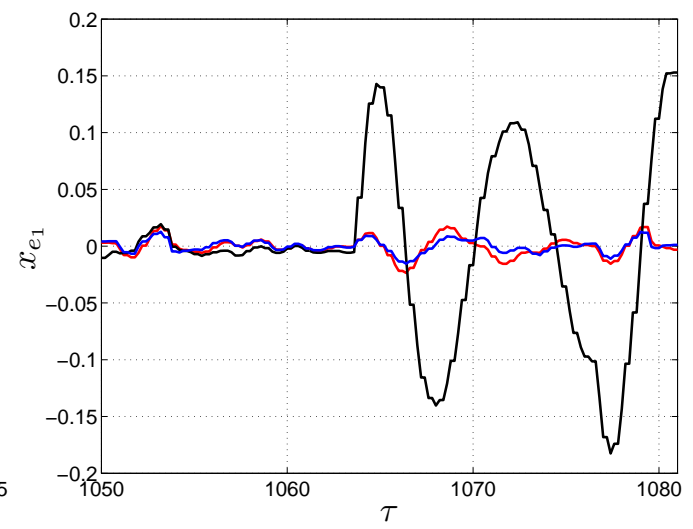

(b)

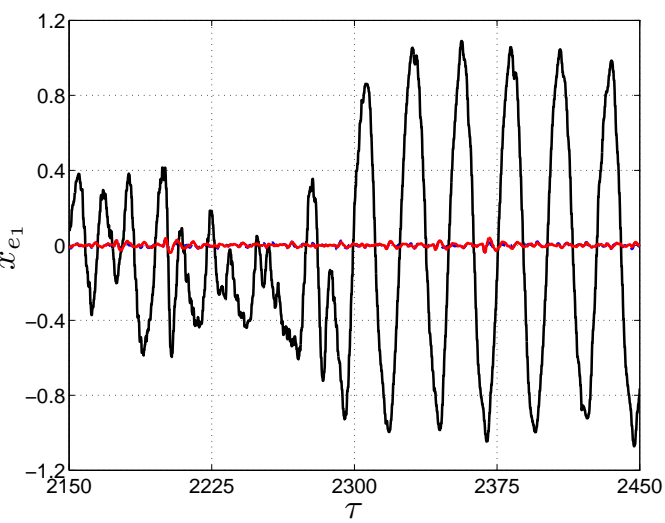

(d)

Figure 11: Tracking performance of the classical MCS and those of the MCSI-PP and the $\sigma$-MCSI. MCS (black line); MCSI-PP (red line) and $\sigma$-MCSI (blue line), reference trajectory (orange line). (a) Tracking performance of all the controllers during the onset of instability with the MCS and (b) related tracking errors. (c) Tracking performance of all the controllers when the classical MCS shows lack of robustness, and (d) related tracking errors. 


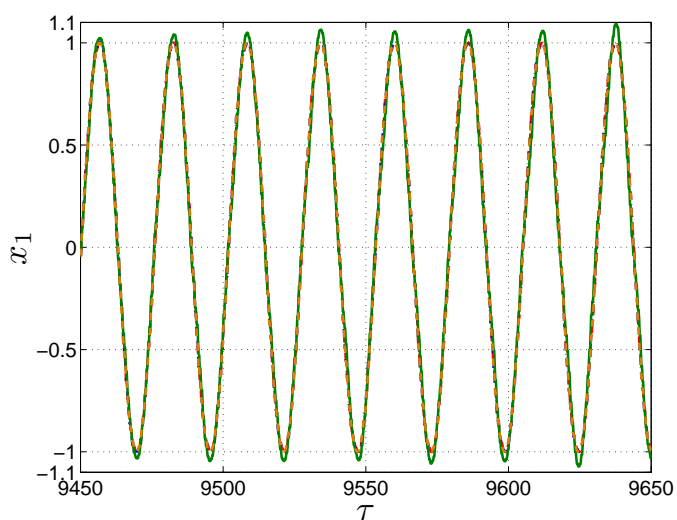

(a)

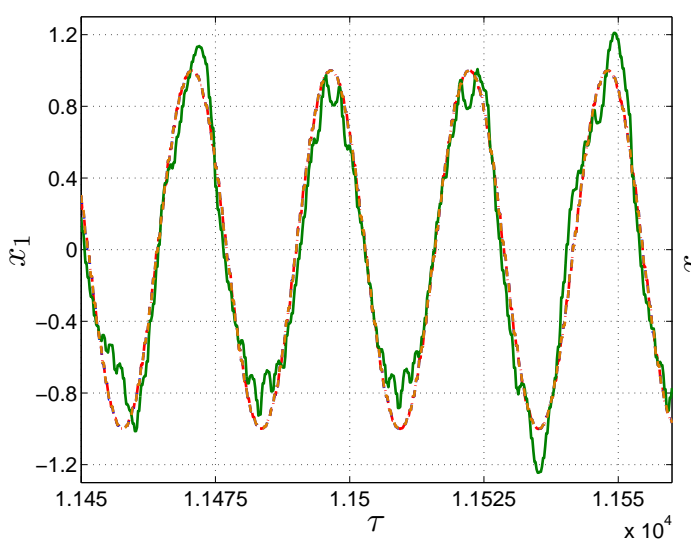

(c)

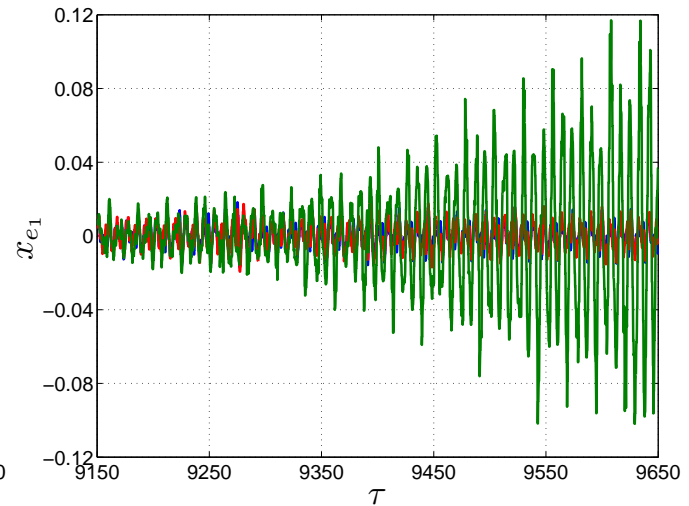

(b)

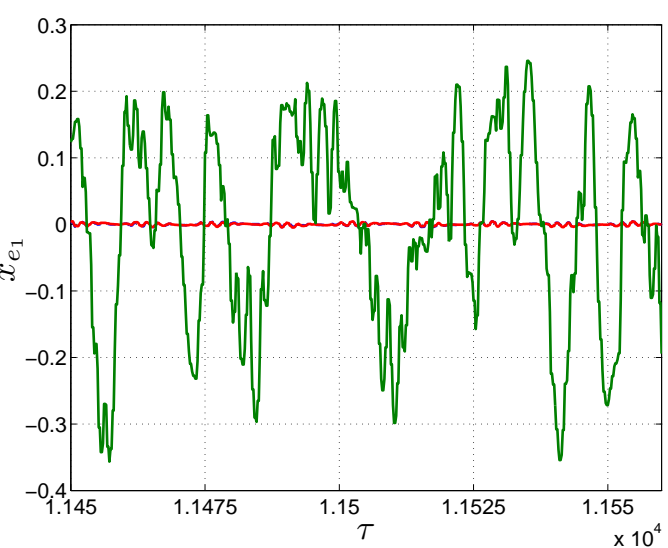

(d)

Figure 12: Tracking performance of the classical MCSI and those of the MCSI-PP and the $\sigma$-MCSI. MCSI (green line); MCSI-PP (red line) and $\sigma$-MCSI (blue line), reference trajectory (orange line). (a) Tracking performance of all the controllers during the onset of instability with the MCSI and (b) related tracking errors. (c) Tracking performance of all the controllers when the classical MCSI shows lack of robustness, and (d) related tracking errors. 


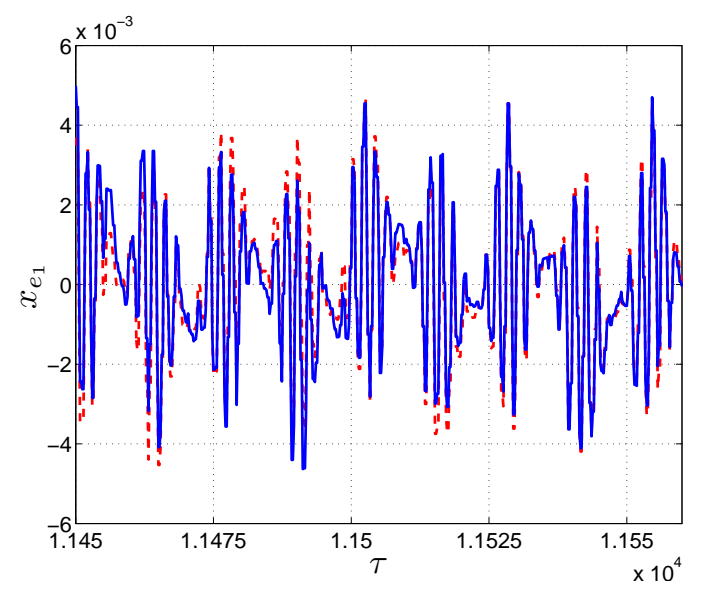

Figure 13: Tracking error when the minimal control synthesis algorithms embed locking methods, MCSI-PP (red line) and $\sigma$-MCSI (blue line).

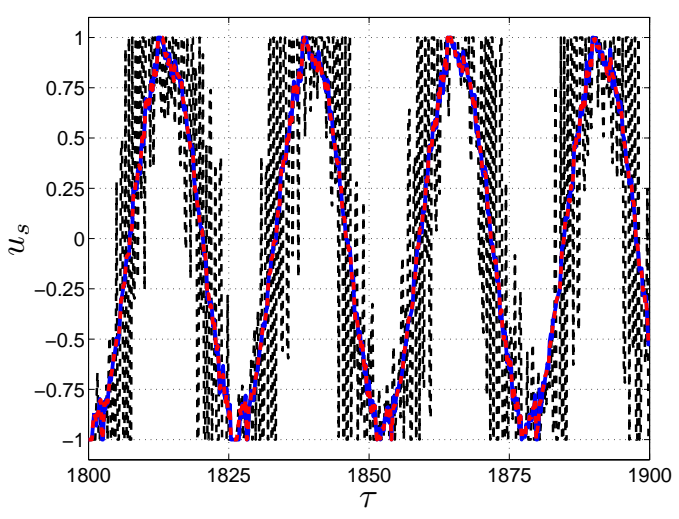

(a)

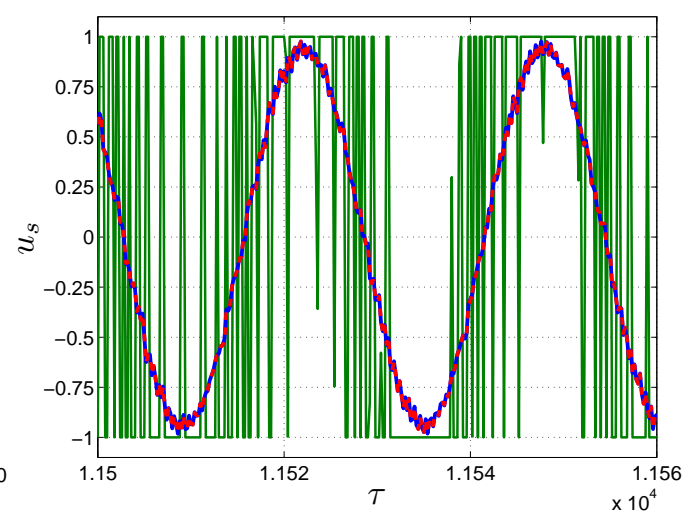

(b)

Figure 14: Adaptive control actions (a) MCS (black line), MCSI-PP (red line) and $\sigma$-MCS (blue line); (b) MCSI (green line), MCSI-PP (red line) and $\sigma$-MCS (blue line). 
preassigned set, thus extending to the continuous-time case the results recently presented for discrete-time systems in [1]. Instead, the latter approach allows to bound the drift of the MCS gains while ensuring global uniform ultimate boundedness in the face of persistent $L_{\infty}$ disturbances. In addition, when $\sigma$-modification is adopted, a bound for the closed-loop tracking error is explicitly computed as a function of the $L_{\infty}$-norm of the disturbance. We note that for the novel MCSI strategies not only methods for locking adaptive gains have been designed, but the same strategies have been applied for also to guarantee boundedness of the integral control when there exists a residual tracking error.

The effectiveness of the control schemes to limit the magnitude of the adaptive gains and, at the same time, guarantee tracking performance of the reference dynamics was shown both analytically, by using a Lyapunov approach, and numerically, by means of a set of representative examples which included the control of a power electronics converter as an engineering case study. Finally, closed-loop performance of the control schemes embedding locking strategies were compared to those provided by the classical MCS algorithms to further confirm that bounded adaptive gains can be achieved without a loss of tracking performance.

As future work, the approaches here presented to avoid unbounded drifts of the control gains will be extended to the novel adaptive control schemes for the control of piecewise affine systems with unknown parameters recently proposed in the literature $[26,41]$. In so doing, the robustness of the proposed adaptive methods for this class of discontinuous systems in the face of bounded disturbances will be systematically studied.

\section{Appendix A. Proof of Lemma 1}

The proof follows similarly to that given in [2].

Let us first recall the scalar functions $h_{X_{j}}, h_{I_{j}}, j=1, \ldots, n$, and $h_{R}$, defined in (18), and consider the vector function

$$
h^{T}=\left[\begin{array}{llll}
h_{1} & h_{2} & \ldots & h_{2 n+1}
\end{array}\right]=\left[\begin{array}{lllll}
-h_{X}^{T} & \vdots & -h_{R} & \vdots & h_{I}^{T}
\end{array}\right] .
$$

Notice that

$$
\phi_{e}^{T} \Gamma_{\alpha}^{-1} f=\left(\phi^{\star}-\phi\right)^{T} \Gamma_{\alpha}^{-1} f=\sum_{j=1}^{2 n+1} \frac{1}{\widehat{\alpha}_{j}}\left(\phi_{j}^{\star}-\phi_{j}\right) f_{j} .
$$

As $\widehat{\alpha}_{j}>0, j=1, \ldots, 2 n+1$, it suffices to see that $\left(\phi_{j}^{\star}-\phi_{j}\right) f_{j} \geq 0, j=$ 
$1, \ldots, 2 n+1$, for any possible situation. This has to take into account that $\phi\left(t_{0}\right) \in \Lambda_{\phi}$, which guarantees $\phi \in \Lambda_{\phi}$ as well because of the parameter projection algorithm (18).

i) If $\phi_{j} \in\left(\phi_{j}^{l}, \phi_{j}^{l}\right)$, or $\phi_{j}=\phi_{j}^{l}$ and $h_{j} \geq 0$, or $\phi_{j}=\phi_{j}^{u}$ and $h_{j} \leq 0$, then $f_{j}=0$; therefore, $\left(\phi_{j}^{\star}-\phi_{j}\right) f_{j}=0$.

ii) If $\phi_{j}=\phi_{j}^{l}$ and $h_{j}<0$, then $\phi_{j}^{\star}-\phi_{j}>0$ and $f_{j}=-h_{j}>0$; therefore, $\left(\phi_{j}^{\star}-\phi_{j}\right) f_{j}>0$.

iii) If $\phi_{j}=\phi_{j}^{u}$ and $h_{j}>0$, then $\phi_{j}^{\star}-\phi_{j}<0$ and $f_{j}=-h_{j}<0$; therefore, $\left(\phi_{j}^{\star}-\phi_{j}\right) f_{j}>0$.

\section{Appendix B. Proof of Lemma 2}

The proof extends the one presented in [2], as in this case we take into account the presence of the matrix $\Gamma_{\alpha}^{-1} \Gamma_{\rho}$.

Using (17) and (22) one has that

$$
\phi_{e}^{T} \Gamma_{\alpha}^{-1} f=-\sigma_{\phi}(\|\phi\|) \phi_{e}^{T} \Gamma_{\alpha}^{-1} \Gamma_{\rho} \phi .
$$

From the definition of $\sigma_{\phi}(\|\phi\|)$ in (23) it is $\sigma_{\phi}(\|\phi\|)=0$ for $\|\phi\| \leq \widehat{\mathcal{M}}_{\phi}$, so the Lemma is trivially fulfilled in this case. Moreover, one can easily check that

$$
\begin{aligned}
-\sigma_{\phi}(\|\phi\|) \phi_{e}^{T} \Gamma_{\alpha}^{-1} \Gamma_{\rho} \phi & =\sigma_{\phi}(\|\phi\|)\left(\phi-\phi^{\star}\right)^{T} \Gamma_{\alpha}^{-1} \Gamma_{\rho} \phi= \\
& =\frac{1}{2} \sigma_{\phi}(\|\phi\|)\left(\phi^{T} \Gamma_{\alpha}^{-1} \Gamma_{\rho} \phi-\phi^{\star T} \Gamma_{\alpha}^{-1} \Gamma_{\rho} \phi^{\star}+\phi_{e}^{T} \Gamma_{\alpha}^{-1} \Gamma_{\rho} \phi_{e}\right),
\end{aligned}
$$

i.e.

$$
\begin{aligned}
\phi_{e}^{T} \Gamma_{\alpha}^{-1} f & =\frac{1}{2} \sigma_{\phi}(\|\phi\|)\left(\mathcal{A}_{1}+\mathcal{A}_{2}\right), \\
\mathcal{A}_{1} & =\phi^{T} \Gamma_{\alpha}^{-1} \Gamma_{\rho} \phi-\phi^{\star T} \Gamma_{\alpha}^{-1} \Gamma_{\rho} \phi^{\star} \\
\mathcal{A}_{2} & =\phi_{e}^{T} \Gamma_{\alpha}^{-1} \Gamma_{\rho} \phi_{e} .
\end{aligned}
$$

The term $\mathcal{A}_{2}$ is non-negative by construction for all $\phi, \phi^{\star}$. In turn, for $\mathcal{A}_{1}$ we have that

$$
\mathcal{A}_{1}=\phi^{T} \Gamma_{\alpha}^{-1} \Gamma_{\rho} \phi-\phi^{\star T} \Gamma_{\alpha}^{-1} \Gamma_{\rho} \phi^{\star} \geq \lambda_{\min }\left(\Gamma_{\alpha}^{-1} \Gamma_{\rho}\right)\|\phi\|^{2}-\lambda_{\max }\left(\Gamma_{\alpha}^{-1} \Gamma_{\rho}\right)\left\|\phi^{\star}\right\|^{2} .
$$


Recalling now the definition of $\widehat{\mathcal{M}}$ in (24), it is immediate that $\mathcal{A}_{1}>0$ for all $\phi>\widehat{\mathcal{M}}$. As also $\sigma_{\phi}(\|\phi\|)>0$ for all $\phi>\widehat{\mathcal{M}}$, it results that $\phi_{e}^{T} \Gamma_{\alpha}^{-1} f>0$ for all $\phi>\widehat{\mathcal{M}}$.

Finally, when $\phi \geq 2 \widehat{\mathcal{M}}_{\phi},(23),($ B.1) and (B.2) yield

$$
\phi_{e}^{T} \Gamma_{\alpha}^{-1} f=\frac{\eta_{\phi}}{2}\left(\mathcal{A}_{1}+\mathcal{A}_{2}\right)>\frac{\eta_{\phi}}{2} \mathcal{A}_{2}=\frac{\eta_{\phi}}{2} \phi_{e}^{T} \Gamma_{\rho} \Gamma_{\alpha}^{-1} \phi_{e}
$$

Hence, (45) is verified as well.

\section{Acknowledgements}

J.M. Olm was partially supported by the spanish Ministerio de Educación project DPI2013-41224-P and the catalan AGAUR project 2014 SGR 267.

\section{References}

[1] U. Montanaro, J. M. Olm, Discrete-time integral MRAC with minimal controller synthesis and parameter projection, J. Franklin Institute 352 (12) (2015) 5415-5436.

[2] G. Tao, Adaptive Control Design and Analysis, John Wiley \& Sons, Inc. Hoboken, NJ, 2003.

[3] Y. Yang, X. Chen, C. Li, Transient performance improvement in model reference adaptive control using image optimal method, J. Franklin Institute 352 (1) (2015) 16-32.

[4] R. Ashok, Y. Shtessel, Control of fuel cell-based electric power system using adaptive sliding mode control and observation techniques, J. Franklin Institute 352 (11) (2015) 4911-4934.

[5] Y. Yang, C. Ge, H. Wang, X. Li, C. Hua, Adaptive neural network based prescribed performance control for teleoperation system under input saturation, J. Franklin Institute 352 (5) (2015) 1850-1866.

[6] R. Chi, Z. Hou, S. Jin, A data-driven adaptive ILC for a class of nonlinear discrete-time systems with random initial states and iteration-varying target trajectory, J. Franklin Institute 352 (6) (2015) 2407-2424. 
[7] Y. Li, S. Tong, T. Li, Adaptive fuzzy output feedback dynamic surface control of interconnected nonlinear pure-feedback systems, IEEE Trans. Cybernetics 45 (1) (2015) 138-149.

[8] Y. Li, S. Tong, T. Li, Composite adaptive fuzzy output feedback control design for uncertain nonlinear strict-feedback systems with input saturation, IEEE Trans. Cybernetics 45 (10) (2015) 2299-2308.

[9] Y. Li, S. Tong, Adaptive fuzzy output-feedback stabilization control for a class of switched non-strict-feedback nonlinear systems, IEEE Trans. Cybernetics, in press.

[10] D. Stoten, H. Benchoubane, Empirical studies of an MRAC algorithm with minimal controller synthesis, Internat. J. Control 51 (4) (1990) 823-849.

[11] D. Stoten, H. Benchoubane, Robustness of minimal controller synthesis algorithm, Internat. J. Control 51 (4) (1990) 851-861.

[12] M. di Bernardo, A. di Gaeta, U. Montanaro, S. Santini, Synthesis and experimental validation of the novel LQ-NEMCSI adaptive strategy on an electronic throttle valve, IEEE Trans. Control Systems Technology 18 (6) (2010) 1325-1337.

[13] M. di Bernardo, A. di Gaeta, U. Montanaro, J. Olm, S. Santini, Discretetime mrac with minimal controller synthesis of an electronic throttle body, in: Proc. 18th IFAC World Congress, 2011, pp. 5064-5069.

[14] U. Montanaro, A. di Gaeta, V. Giglio, Adaptive tracking control of a common rail injection system for gasoline engines: a discrete-time integral minimal control synthesis approach, IEEE Trans. Control Systems Technology 21 (5) (2013) 1940-1948.

[15] U. Montanaro, A. di Gaeta, V. Giglio, An MRAC approach for tracking and ripple attenuation of the common rail pressure for GDI engines, in: Proc. 18th IFAC World Congress, 2011, pp. 4173-4180.

[16] A. di Gaeta, C. Hoyos-Velasco, U. Montanaro, Cycle-by-cycle adaptive force compensation for the soft-landing control of an electro-mechanical engine valve actuator, Asian J. Control 17 (5) (2015) 1707-1724. 
[17] D. Stoten, M. di Bernardo, Application of the minimal control synthesis algorithm to the control and synchronization of chaotic systems, Internat. J. Control 65 (6) (1996) 925-938.

[18] D. Stoten, E. Gómez, Adaptive control of shaking tables using the minimal controller synthesis algorithm, Philosophical Trans. Royal Soc. London 357 (9) (2001) 1697-1723.

[19] A. Hillis, A. Harrison, D. Stoten, A comparison of two adaptive algorithms for the control of active engine mounts, J. Sound Vibration 286 (1-2) (2005) $37-54$.

[20] A. Gizatullin, K. Edge, Adaptive control for a multi-axis hydraulic test rig, Proc. Inst. of Mechanical Eng. - Part I: J. Systems and Control Eng. 221 (2) (2007) 183-198.

[21] L. Rossi, A. Irace, U. Montanaro, M. di Bernardo, G. Breglio, Structural vibration control of a cantilever beam by MRAC method, in: Proc. 2nd Internat. Symp. Reliability of Optoelectronics For Space, Cagliari, Italy, 2010.

[22] H. Benchoubane, D. Stoten, The decentralized minimal controller synthesis algorithm, Internat. J. Control 56 (4) (1992) 967-983.

[23] D. Stoten, H. Benchoubane, The extended minimal controller sysnthesis algorithm, Internat. J. Control 56 (5) (1992) 1139 - 1165.

[24] D. Stoten, S. Neild, The error-based minimal control synthesis algorithm with integral action, Proc. Inst. of Mechanical Eng. - Part I: J. Systems and Control Eng. 217 (3) (2003) 187-201.

[25] M. di Bernardo, U. Montanaro, S. Santini, Minimal control synthesis adaptive control of continuous bimodal piecewise affine systems, SIAM J. Control and Optimization 48 (7) (2010) 4242-4261.

[26] M. di Bernardo, U. Montanaro, S. Santini, Hybrid model reference adaptive control of piecewise affine systems, IEEE Trans. Automatic Control 58 (2) (2013) 304-316.

[27] M. di Bernardo, C. Hoyos-Velasco, U. Montanaro, S. Santini, Experimental implementation and validation of a novel minimal control synthesis adaptive controller for continuous bimodal piecewise affine systems, Control Eng. Practice 20 (3) (2012) 269-281. 
[28] M. di Bernardo, F. di Gennaro, J. M. Olm, S. Santini, Discrete-time minimal control synthesis adaptive algorithm, Internat. J. Control 83 (4) (2010) 26412657.

[29] M. di Bernardo, A. di Gaeta, U. Montanaro, J. M. Olm, S. Santini, Experimental validation of the discrete-time MCS adaptive strategy, Control Eng. Practice 21 (6) (2013) 847-859.

[30] M. di Bernardo, U. Montanaro, J. Olm, S. Santini, Model reference adaptive control of discrete-time piecewise linear systems, Internat. J. Robust Nonlinear Control 23 (7) (2013) 709-730.

[31] U. Montanaro, A. di Gaeta, V. Giglio, Robust discrete-time MRAC with minimal controller synthesis of an electronic throttle body, IEEE/ASME Trans. Mechatronics 19 (2) (2014) $524-537$.

[32] B. Anderson, R. Bitmead, C. Johnson, P. Kokotovic, R. Kosut, I. Mareels, L. Praly, B. Riedle, Stability of adaptive systems: passivity and averaging analysis, The M.I.T. Press, Cambridge, MA and London, 1986.

[33] S. Sebusang, D. Stoten, Controller gain bounding in the minimal control synthesis algorithm, in: Proc. 30th Southeastern Symp. on System Theory, 1998, pp. 141-145.

[34] H. Khalil, Nonlinear Systems, 3rd Edition, Prentice Hall, 2002.

[35] N. Mohan, T. Undeland, W. Robbins, Power Electronics: Converters, Applications, and Design, 3rd Edition, Cambridge University Press, 2002.

[36] S. Hodgson, D. Stoten, Passivity-based analysis of the minimal control synthesis algorithm, Internat. J. Control 63 (1) (1996) 67-84.

[37] D. Stoten, The adaptive minimal control synthesis algorithm with integral action, in: Proc. 21st Internat. Conf. Industrial Electronics, Control, and Instrumentation, Vol. 2, 1995, pp. 1646-1651.

[38] A. Salvi, S. Santini, D. Biel, J. Olm, M. di Bernardo, Model reference adaptive control of a full-bridge buck inverter with minimal controller synthesis, in: Proc. IEEE 52nd Conf. Decision and Control, 2012, pp. 3469 - 3474. 
[39] S. Malo, R. Grinó, Adaptive feed-forward cancellation control of a fullbridge dc-ac voltage inverter, in: Proc. 17th IFAC World Congress, 2008, pp. 4571-4576.

[40] O. Bursi, D. Stoten, L. Vulcan, Convergence and frequency-domain analysis of a discrete first-order model reference adaptive controller, Structural Control and Health Monitoring 14 (5) (2007) 777-807.

[41] M. di Bernardo, U. Montanaro, R. Ortega, S. Santini, Extended hybrid model reference adaptive control of piecewise affine systems, Nonlinear Analysis: Hybrid Systems 21 (2016) 11-21. 